\title{
Metabolic reprogramming in chondrocytes to promote mitochondrial respiration reduces downstream features of osteoarthritis
}

\section{Yoshifumi Ohashi}

Department of Orthopedic Surgery, Nagoya University Graduate School of Medicine

Nobunori Takahashi ( $\sim$ nobunori@med.nagoya-u.ac.jp )

Department of Orthopedic Surgery, Nagoya University Graduate School of Medicine

\section{Kenya Terabe}

Department of Orthopedic Surgery, Nagoya University Graduate School of Medicine

\section{Saho Tsuchiya}

Department of Orthopedic Surgery, Japan Community Health care Organization, Tokyo Shinjuku Medical Center

\section{Toshihisa Kojima}

Department of Orthopedic Surgery, Nagoya University Graduate School of Medicine

\section{Cheryl Knudson}

Department of Anatomy and Cell Biology, Brody School of Medicine, East Carolina University

\section{Warren Knudson}

Department of Anatomy and Cell Biology, Brody School of Medicine, East Carolina University

\section{Shiro Imagama}

Department of Orthopedic Surgery, Nagoya University Graduate School of Medicine

\section{Research Article}

Keywords: chondrocytes, Metabolic dysfunction, galactose replacement, osteoarthritis care

Posted Date: December 15th, 2020

DOI: https://doi.org/10.21203/rs.3.rs-113139/v1

License: (c) (i) This work is licensed under a Creative Commons Attribution 4.0 International License. Read Full License

Version of Record: A version of this preprint was published at Scientific Reports on July 23rd, 2021. See the published version at https://doi.org/10.1038/s41598-021-94611-9. 


\section{Abstract}

Metabolic dysfunction in chondrocytes drives the pro-catabolic phenotype associated with osteoarthritic cartilage. In this study, substitution of galactose for glucose in culture media was used to promote a renewed dependence on mitochondrial respiration for ATP production. Galactose replacement alone blocked enhanced usage of the glycolysis pathway by IL $1 \beta$-activated chondrocytes as detected by realtime changes in the rates of proton acidification of the medium and changes in oxygen consumption. The change in mitochondrial activity due to galactose was visualized as a rescue of mitochondrial membrane potential but not an alteration in the number of mitochondria. Galactose-replacement reversed other markers of dysfunctional mitochondrial metabolism, including blocking the production of reactive oxygen species, nitric oxide, and the synthesis of inducible nitric oxide synthase. Of more clinical relevance, galactose-substitution blocked downstream functional features associated with osteoarthritis, including enhanced levels of MMP13 mRNA, MMP13 protein, and the degradative loss of proteoglycan from intact cartilage explants. Blocking baseline and IL1 $\beta$-enhanced MMP13 by galactose-replacement in human osteoarthritic chondrocyte cultures inversely paralleled increases in markers associated with mitochondrial recovery, phospho-AMPK, and PGC1a. Comparisons were made between galactose replacement and the glycolysis inhibitor 2-deoxyglucose. Targeting intermediary metabolism may provide a novel approach to osteoarthritis care.

\section{Introduction}

Previous studies have suggested that the degradative features associated with osteoarthritis (OA) in articular cartilage are the downstream result of changes in the metabolism of resident chondrocytes ${ }^{1,2}$. These OA features include enhanced production of extracellular proteinases such as MMPs, ADAMTS4/5, the resultant loss of proteoglycan, and eventual breakdown of the collagenous network. Changes in metabolism associated with OA chondrocytes include enhanced dependence on glycolysis for cellular ATP production, coordination with a decrease in mitochondrial respiration, and use of the TCA cycle ${ }^{2}$. This alteration has many features in common with the Warburg Effect of many cancers ${ }^{3}$. We have recently gained insight into the features of these metabolic changes in chondrocytes and how they can be reversed ${ }^{1,2}$.

Initially, we observed that enhanced local synthesis of hyaluronan (HA) via viral overexpression of $\mathrm{HA}$ synthase-2 (HAS2-OE) blocked and reversed the pro-catabolic features of normal chondrocytes activated by IL-1 $\beta$, TNFa or LPS as well as human OA-derived chondrocytes ${ }^{1}$. Surprisingly, however, this was not due to enhanced accumulation of extracellular HA. Rather, viral driven enhancement of HA polysaccharide synthesis altered the intermediary metabolism of the activated chondrocytes due to overusage of intracellular UDP-sugar precursor pools. Similar results were obtained by the treatment of chondrocytes with 4-methylumbelliferone (4MU), a known inhibitor of HA that acts in part by sequestration and depletion of UDP-glucuronic acid ${ }^{4}$. HAS2-OE and $4 \mathrm{MU}$ both cause a reduction in the enhanced usage of the glycolysis pathway by activated, pro-catabolic chondrocytes. The HAS2-OE 
chondroprotective effect can also be mimicked by treatment of activated chondrocytes with 2-deoxyglucose (2-DG) or dichloroacetate (DCA), two glycolysis inhibitors often used to block enhanced glycolysis in tumor cells ${ }^{2}$. Interestingly, in all of these treatments, not only was glycolysis reduced, but ATP production via the TCA cycle was enhanced. This paper seeks to explore how these two metabolic pathways are coupled and which events are critical to the chondroprotective effects.

This study investigated the effects of total glucose replacement with the monosaccharide isomer galactose. Galactose substitution is often used in studies on the Warburg effect observed in tumor cells to tease out the downstream effects of metabolic dysfunction. Galactose substitution is a known activator of the TCA cycle coupled with suppression of glycolysis for the production of ATP. Galactose is metabolized via the Leloir pathway ${ }^{5-7}$ with the conversion of galactose into glucose-6-phosphate (G6P), which then can be used for the glycolysis pathway. Typically, however, the generation of G6P by the Leloir pathway is too slow to meet the energy demands of cells, especially cells, highly dependent on glycolysis. This, in turn, induces a strong compensatory activation of mitochondrial respiration, at least in cells that retain the capacity to adapt and rescue mitochondrial function. For example, healthy cells adapt and survive in either glucose or galactose culture media, whereas many tumor cells with permanentlyimpaired mitochondrial function fail to survive ${ }^{8}$. Galactose substitution also provides an improved approach to complete or partial shutdown of glycolysis by glucose deprivation alone-a condition that often induces cell death ${ }^{9}$.

\section{Results}

Galactose suppresses glycolysis and enhances mitochondrial respiration. Bovine chondrocytes were analyzed in real-time for changes in the rate of $\mathrm{H}^{+}$proton efflux into the culture medium (PER) indicative of glycolytic metabolism. Raw data in Fig. 1A shows changes PER for each culture condition, that occur following injections of select glycolysis inhibitors at 18 and 36 min during the rate assay-inhibitors that define the pathways responsible for the proton release. Calculations were made from such plots from multiple experiments to quantify changes in basal glycolysis (Fig. 1B). Following treatment with IL-1 $\beta$, PER became substantially elevated (Fig. 1A, 1B). However, if chondrocytes were cultured in galactosereplaced medium, this elevation was not only blocked but PER was reduced to levels lower than control cells. Using a mitochondrial stress assay, real times changes in oxygen consumption rate (OCR) were obtained (Fig. 1C, 1D). Raw data (Fig. 1C) include injections of TCA cycle inhibitors at 18 and 54 minutes were used to define mitochondrial contribution to OCR; with data summarized in Fig. 1D. Treatment of chondrocytes with IL-1 $\beta$ resulted in a prominent reduction in mitochondrial respiration as compared to control chondrocytes (Fig. 1C, 1D). Culture of chondrocytes treated with IL-1 $\beta$ in galactose-replaced medium did not exhibit a diminution of OCR. Moreover, OCR levels of chondrocytes in galactose-replaced medium in the absence of IL-1 $\beta$ were significantly enhanced as compared to control chondrocytes in glucose-enriched medium. 
Another test performed using the Sea Horse flux analyzer, termed a Real-Time ATP rate assay, generated similar PER and OCR data to define the pathway contributions to ATP production (in pmol/min ATP). These data are summarized in the stacked bar plot shown in Fig. 2. Control chondrocytes utilized glycolysis to generate approximately $75 \%$ of their ATP (Fig. 2, grey bar); with $25 \%$ obtained from mitochondrial respiration (Fig. 2, white bar). After treatment with IL-1 3 , chondrocytes became more dependent on glycolysis as the source of cellular ATP, with only a small percentage coming from mitochondrial respiration. However, when these same conditions were analyzed in galactose-replaced medium the metabolic changes due to IL-1 $\beta$ were blocked. Interestingly, under the galactose-replacement conditions, ATP production due to mitochondrial respiration, in both control and IL-1 $\beta$ treated cultures, was enhanced with glycolysis now providing only a minor contribution.

Effects of IL-1 $\beta$ and galactose mitochondrial integrity and function. Given the effects of IL-1 $\beta$ and galactose on mitochondrial production of ATP in chondrocytes, we next determined whether this was due to an increase in number of mitochondria or their functional integrity. The red fluorescent tetramethyl rhodamine (TMRM) reagent accumulates in active mitochondria with an intact membrane potential (Fig. 3A) whereas the reagent Mito Tracker Green FM reagent (Mito Green) reacts with free cysteine thiol groups of mitochondrial proteins and identifies all mitochondria. Stained control chondrocytes exhibited close overlap of green and red fluorescence suggesting that nearly all mitochondria are active (Fig. 3A). When the cells were treated with an inhibitor of oxidative phosphorylation, carbonyl cyanide-4phenylhydrazone (FCCP), the mitochondrial membrane potential collapsed, TMRM became diffusely distributed throughout the cytosol while the green fluorescence remained intact. A ratio of TMRM / Mito Green fluorescence intensity was used to quantify these changes (Fig. 3B).

Chondrocytes incubated with IL-1 $1 \beta$ readily lost significant levels of TMRM fluorescence as compared to control chondrocytes (Fig. 3C). However, in chondrocytes cultured in galactose-replaced medium, the IL$1 \beta$-induced reduction in TMRM accumulation was blocked (Fig. 3C, 3D). Moreover, the TMRM / Mito Green ratios of chondrocytes in galactose-replaced medium were higher than those of control chondrocytes (Fig. 3C, 3D). A similar observation was made when chondrocytes were treated with an inhibitor of the glycolysis pathway, 2-deoxyglucose (2-DG). 2-DG (in galactose-containing medium) also blocked the accumulation of TMRM following treatment with IL-1 $\beta$ (Fig. 3C, 3E). However, with the addition of 2-DG, the TMRM / Mito Green ratios remained equivalent to control chondrocytes.

When Mito Green fluorescence was quantified separately, no significant changes were observed under any test conditions of culture (Fig. 3F) suggesting that changes in mitochondrial ATP shown in Fig. 2 were not due to changes in the number of mitochondria per cell.

Galactose blocks markers of mitochondrial damage. Reactive oxygen species (ROS) are well-known byproducts of damaged mitochondria, especially in pro-catabolically activated chondrocytes ${ }^{10}$. ROS were detected in live cells using a cell-permeant green fluorescent reagent, 2,7-dichlorofluorescein diacetate (DCFDA). In control chondrocytes, a few green fluorescent-positive cells were observed scattered throughout various fields of view (Fig. 4A); intensity values quantified in Fig. 4B. As expected, treatment 
with IL1 $\beta$ dramatically increased the number of green fluorescent-positive cells. This increase in ROS was significantly blocked in chondrocytes treated with IL1 $\beta$ under galactose-replaced medium culture conditions (Fig. 4A, 4B). These results were compared to the second series of experiments where chondrocytes were treated with or without IL1 $\beta$ and, with or without the glycolysis inhibitor 2-DG (Fig. 4C, 4D). Again, IL1 $\beta$ treatment caused a pronounced increase in ROS that was blocked by the presence of 2DG.

Nitric oxide (NO), is another oxygen species that is altered in OA and pro-catabolically activated chondrocytes and; is one of the causes of damage to the mitochondrial electron transport system ${ }^{11}$. First, the expression of inducible nitric oxide synthase (iNOS) mRNA was examined by qRT-PCR. As expected, IL1 $\beta$ increased of iNOS mRNA relative to the expression in control chondrocytes (Fig. 5A). However, when chondrocytes were grown under galactose-replaced medium conditions, the increase in iNOS mRNA expression was blocked and, reduced below control baseline levels. iNOS mRNA levels in control chondrocytes grown in galactose-replaced medium were also $\sim 2$-fold lower than control chondrocytes grown in glucose-rich medium. In a separate series of experiments, the glycolysis inhibitor, 2-DG also blocked IL1 $\beta$ induced increases in iNOS mRNA (data not shown). Changes in NO itself were also monitored (Fig. 5B). Following the trend in iNOS mRNA, treatment of chondrocytes with IL1 $\beta$ generated a pronounced increase in NO gas as compared to control chondrocytes (Fig. 5B). This IL1 $\beta$ induced increase in NO did not occur when the chondrocytes were cultured under galactose-replaced medium conditions. Again, in a separate series of experiments, the glycolysis inhibitor, 2-DG, also blocked IL1 $\beta$ induced increases in NO (data not shown).

As a third approach, the Sea Horse flux analyzer was used to measure and quantify in real-time nonmitochondrial oxygen consumption (NMOC); representing oxygen consumption that contributes to the production of both $\mathrm{NO}$ and ROS ${ }^{10,12}$. NMOC is measured after completely blocking mitochondrial respiration using a combination of oxidative phosphorylation inhibitors: Oligomycin A, FCCP, Rotenone, and Antimycin A. In glucose-rich medium conditions, IL $1 \beta$ treatment resulted in an increase in NMOCrelated OCR (oxygen consumption rate) above that of control chondrocytes (Fig. 5C). However, in galactose-replaced medium this increase in NMOC-related OCR was blocked. Treatment of chondrocytes with IL1 $\beta$ in the presence of 2-DG resulted in a similar block of IL1 $\beta$-induced increase (data not shown).

These results suggest that galactose-replaced medium blocks the production and accumulation of oxygen by-products highly associated with mitochondrial damage.

\section{Galactose-replaced culture conditions block pro-catabolic features of activated chondrocytes and} cartilage. Galactose appears to block damage and rescue functional integrity of mitochondria in chondrocytes treated with IL1 $\beta$. To determine whether galactose-mediated rescue of mitochondria translated into effects related to $O A$, an examination was made of two markers of the OA phenotype, namely, expression of the collagenase MMP-13 and the loss of proteoglycan (measured as sulfated glycosaminoglycan loss) from explants of intact cartilage tissue. Chondrocytes treated with IL-1 $\beta$ cultured in glucose-rich medium exhibited a pronounced increase in MMP-13 mRNA expression as 
compared to control cells (Fig. 6A). When the chondrocytes were cultured in galactose-replaced medium, this increase in MMP-13 mRNA was blocked (Fig. 6A). Moreover, the MMP-13 mRNA levels in chondrocytes in galactose-replaced medium, with or without IL1 $\beta$, were both lower than control chondrocytes cultured in glucose-rich medium.

Next, explants of intact bovine cartilage were cultured in glucose or galactose medium. After 1 week in culture, sections of control cartilage stained bright red with safranin 0 , indicative of a rich proteoglycan distribution throughout the cartilage matrix (Fig. 6C). After treatment with IL1 $\beta$, much of the red safranin $O$ staining was lost from the tissue-an event that mimics the loss of proteoglycan (an important diagnostic feature) in human OA. The proteoglycan lost from the tissue can be detected in the medium by use of a colorimetric assay for sulfated glycosaminoglycan as shown in Fig. 6B. When the cartilage explants were cultured in galactose-replaced medium, no IL1 $\beta$-induced loss of safranin 0 staining (Fig. 6C) and no increase in shed degradation products in the medium (Fig. 6B) were observed. Lower background levels of shed glycosaminoglycan were also observed in control explants (without IL1 $\beta$ ) in galactose-replaced medium. It should also be noted that the sections of control cartilage explants grown in galactose-replaced medium appear healthy with rich safranin 0 staining suggesting that galactosereplacement conditions do not exert a deleterious or nutrient-deficiency effect on the tissue even after 7 days of culture.

Effects of IL-1 $\beta$ and galactose on human OA chondrocytes. The effects of IL-1 $\beta$ and galactosereplacement on mitochondrial function were next tested on high-density cultures of human articular chondrocytes-cells freshly derived from human OA patient knee cartilage. For these chondrocytes, protein lysates were prepared and analyzed for the expression of key proteins related to control of mitochondrial respiration [namely, p-AMPK and PGC1a (3)] and MMP-13, a protein marker indicative of changes in the pro-catabolic state of the cells ${ }^{13}$. IL-1 $\beta$ significantly enhanced the expression of MMP-13 protein in human OA chondrocytes cultured in glucose-rich medium. However, this enhancement in MMP13 protein due to IL-1 $\beta$ was blocked in chondrocytes cultured in galactose-replaced medium. Also, background levels of MMP-13 in control chondrocytes (grown in glucose medium) were reduced when the cells were grown in galactose-replaced medium (Fig. 7A, B). These results were similar to the effects of galactose on bovine MMP-13 mRNA shown in Fig. 6A. Next, the same lysates were examined for the expression of proteins that regulate mitochondrial function. As shown in Fig. 7C and 7D, control and IL-1 $\beta$ treated human OA chondrocytes in glucose-based medium exhibited barely detectable levels of p-AMPK. However, when the control OA chondrocytes were cultured instead in medium replaced with galactose, substantially enhanced levels of $\mathrm{p}$-AMPK were observed. Moreover, these enhanced $\mathrm{p}$-AMPK levels were not diminished when the chondrocytes were treated with IL-1 $\beta$ in galactose-replaced medium (Fig. 7C, 7D). Treatment of chondrocytes with IL1 $\beta$ in the presence of 2-DG resulted in a similar change of IL1 $\beta$ induced decrease (data not shown).

The same trend was observed in the expression of another mitochondrial regulated protein activated downstream of p-AMPK termed PGC1a (peroxisome proliferator-activated receptor-y coactivator 1-a) ${ }^{14}$. Like p-AMPK expression, enhanced PGC1a was observed in human OA chondrocytes cultured under 
galactose-replaced medium conditions. Again, little diminishment of PGC1a was observed following the addition of IL-1 $\beta$ in galactose-replaced medium (Fig. 7E, 7F).

\section{Discussion}

In this study, we observed that chondrocytes, when cultured in a medium depleted of all glucose and replaced with galactose, underwent a metabolic reprogramming. Control chondrocytes in glucose-rich medium exhibited a high dependence on the glycolysis pathway for ATP production, a dependence that became more prominent when the cells were pro-catabolically activated with IL-1 $\beta$ treatment. This dependence on glycolysis was inversely echoed by a deficit in ATP produced through mitochondrial respiration. As such, these conditions in glucose-rich medium mimic the mitochondrial dysfunction associated with $\mathrm{OA}^{2,14-18}$. In a galactose-replaced medium, the use of glycolysis by chondrocytes was substantially reduced; mitochondrial respiration increased concomitant with an increase in overall mitochondrial function. More importantly, the development of pro-catabolic features following IL-1 $\beta$ treatment, including MMP13 production and proteoglycan loss from cartilage explants, were blocked within vitro culture in galactose-replaced media.

Previously we reported that the overexpression of hyaluronan synthase-2 (HAS2-OE) blocked MMP13 and MMP3 synthesis in activated chondrocytes but by way of intracellular changes and not feedback due to the accumulation of extracellular hyaluronan $(\mathrm{HA})^{1}$. Treatment of activated chondrocytes with the HA inhibitor $4 \mathrm{MU}$ also blocked the pro-catabolic response ${ }^{4}$. Given that both seemingly opposing treatments had in common, the depleted UDP-glucuronic acid pools (via hyaluronan biosynthesis or substrate sequestration) led us to examine potential changes in overall chondrocyte metabolism. Enhanced HA production, as well as $\mathrm{HA}$ inhibition by $4 \mathrm{MU}$, both reduced the use of glycolysis in activated chondrocytes and rescued IL1 $\beta$-induced deficits in mitochondrial respiration ${ }^{2}$. These metabolic changes were coincident with a reduction in enzymatic degradation of the extracellular matrix. To further document that the metabolic shifts of HAS2-OE and 4MU were due, in part, to depletion of intracellular precursor pools of glucose derivatives, we examined the low-dose use of inhibitors of the glycolysis pathway, namely 2DG and dichloroacetate (DCA) ${ }^{2}$. As with HAS2-OE and 4MU, both glycolysis inhibitors 2DG and DCA rescued mitochondrial activity in activated chondrocytes, resulting in a downstream inhibition of the OA-like procatabolic phenotype. However, all these experiments were performed in a standard glucose-rich culture medium.

It is known that many cells in glucose-rich culture adapt to the prominent use of the glycolysis pathway, even under aerobic conditions ${ }^{19}$. This has made it difficult to study the role of mitochondria and oxidative phosphorylation. In some cells, this is termed "Crabtree Effect," where there is enhanced use of glycolysis even when mitochondria are still present and functional ${ }^{20}$. Data in Fig. 3 of this paper suggests that functional mitochondria are present in control chondrocytes even though $~ 75 \%$ of ATP was being generated by glycolysis (Fig. 2). To overcome these adaptive changes, investigators have used galactose-replacement. Galactose can be metabolized by the Leloir Pathway to glucose-1-phosphate and 
then enter the glycolysis pathway after modification to glucose-6-phosphate. However, the multi-step conversion of galactose to glucose-6-phosphate is slower than starting with glucose and results in conditions that promote oxidative phosphorylation to generate ATP ${ }^{5}$. Galactose can also be metabolized to glyceraldehyde-3-phosphate and pyruvate through the phosphate pentose shunt but, that process generates no net ATP and thus again forces cells to rely on mitochondrial oxidative phosphorylation for ATP ${ }^{21}$. Fortunately, cultured cells generally grow well under galactose-replacement conditionsconditions that allow for a more accurate evaluation of the role of mitochondria in a host of cellular activities $^{6,8}$.

Using galactose-replacement conditions in our study, we observed that lactate production (measured as the rate of proton acidification of the medium or PER) was highly reduced; OCR (oxygen consumption related to oxidative phosphorylation of the tricarboxylic acid or Kreb's cycle) was very highly enhanced as compared to glucose-rich conditions (Fig. 1). These results provide evidence that control chondrocytes could switch from 75\% (ATP produced from glycolysis) / 25\% (ATP generated from mitochondrial respiration) to $12 \%$ / $88 \%$ in galactose-replaced medium (Fig. 2). Even though the control chondrocytes were highly glycolytic, they exhibited mitochondria with an intact, functional mitochondrial membrane potential (Fig. 3). After treatment with IL-1 $\beta$ in glucose-rich media, the otherwise normal, healthy chondrocytes developed a pro-catabolic phenotype reminiscent of events associated with human OA. Mitochondrial function diminished (but not the number of mitochondria, Fig. 3), nitric oxide (NO), inducible Nitric Oxide synthase (iNOS), and reactive oxygen species (ROS) levels became elevated (Fig. 4). But critically, markers indicative of OA-like destruction of the extracellular matrix, namely MMP13 mRNA and loss of proteoglycan from cartilage explants (indicative of ADAMTS4 activity), were prominently enhanced (Fig. 6). All of these IL1 $\beta$-induced features were blocked when the experiments were performed in parallel in galactose-replaced medium. Mitochondria of IL1 $\beta$-treated chondrocytes in galactose-replaced medium regained membrane potential, pAMPK levels spiked coordinate with decreases in NO, iNOS mRNA, ROS, and NMOC (Figs. 3-5). These data suggest that the induced mitochondrial dysfunction and associated pro-catabolic effect in chondrocytes are reversible similar to the Crabtree Effect observed in several other cell types ${ }^{20}$.

We used IL-1 $1 \beta$ to activate chondrocytes in this study. IL $1 \beta$ is commonly used by many investigators to induce and mimic OA-like features, including changes in metabolism associated with $O A$, such as an increase in NO, leading to mitochondrial damage and release of ROS and the activation of extracellular enzymatic cartilage damage-the major issues of $O A^{22}$. However, the genesis of human $O A$ is more complex and multifaceted. In the natural state, the onset of OA is due to trauma, aging, inflammation, and the effects of feedback by the degradation products of tissue damage (termed DAMPs) ${ }^{15,23-25}$. IL1 $\beta$ may not induce all of the features of human disease, but it is highly reproducible, and since it is used by many investigators, it allows cross-comparisons between studies. In our previous studies, we made comparisons between IL-1 $\beta$ other commonly employed inducers, including; the inflammatory cytokine TNFa, three relevant examples of DAMPS, namely fibronectin fragments, HA oligosaccharides, and LPS 1,2 . Each of these inducers was capable of activating molecular markers and features of OA (such as 
MMP13 and proteoglycan release from cartilage) in bovine or human OA chondrocytes as well as cartilage explants. More importantly, all of these pro-catabolic features were blocked by co-incubation of each inducer and HAS2-OE ${ }^{1}, 4 \mathrm{MU}^{2,4}, 2 \mathrm{DG}$ or $\mathrm{DCA}^{2}{ }^{2}$. In addition, non-induced human chondrocytes derived from human OA patients ${ }^{1,2,4}$ were also examined. Like in this study (Fig. 7), albeit with variability from patient-to-patient, baseline MMP13 was elevated without the need of an inducer such as IL-1 $\beta$. These elevated MMP13 levels could also be reversed by treatment with HAS2-OE, 4MU, 2DG, DCA or, in this case, by galactose-replacement ${ }^{1-3}$. After establishing these results, only IL $1 \beta$ was employed as an inducer in this study.

It may very well be that $\mathrm{OA}$ chondrocytes have undergone long-term metabolic reprogramming and mitochondrial dysfunction, and even depletion of mitochondria. This long-term metabolic reprogramming is the distinguishing feature between the Warburg Effect (long term) and the Crabtree Effect (short term, reversible) in tumor cells ${ }^{20}$. It should be noted that control human OA chondrocytes have enhanced MMP13 protein levels and non-detectable PAMPK at baseline even before treatment with IL1 $\beta$ (Fig. 7). Nonetheless, galactose-replacement substantially reduced both baseline and IL $1 \beta$-activated MMP13 protein levels. Moreover, baseline PAMPK and PGC1a (proteins associated with functional mitochondria) were also substantially enhanced. Thus, although chondrocytes derived from human OA patient tissues may exhibit levels of permanent mitochondrial dysfunction, our galactose-replacement studies suggest that both mitochondrial activity and pro-catabolic activities remain reversible. It is possible that we saw fewer green mitochondria in OA chondrocytes because of autophagy-an attempt by the cells to remove damaged mitochondria to prevent further cellular damage.

There are several limitations to this study. As mentioned above, one limitation of this study was that chondrocytes must be grown in culture to do this kind of study, wherein they readily adapt to a high dependence on glycolysis for ATP. This is typical of many studies on metabolism that require the use of cultured cells, including many tumor cell lines ${ }^{7,8,26}$ and primary cultures such as myotubes ${ }^{6,27}$. This study, like others $7,8,26$ used the galactose-replacement approach to overcome this issue. A second limitation was limited access to human cartilage tissue. As such, studies on human OA cells was limited. It could be concluded that the data presented in Fig. 7 at least matches the same trends in results shown in bovine articular chondrocytes. More work will be done in the future as samples become available. It is recognized that all of this work because it is mechanistic in nature, was performed in vitro. Thus, many questions arise, such as do these chondroprotective results (of glycolysis inhibition/ TCA enhancement) change due to oxygen levels, serum levels, glucose concentrations, cell density used, the timing of experiments, etc. We recently addressed this by examining the effects of $4 \mathrm{MU}$ in an animal (mouse) model of induced $\mathrm{OA}^{28}$. In this study, feeding animals $4 \mathrm{MU}$ provided a protective effect on the development of OA (in vivo) following medial meniscal ligament transection-induced OA. These OA protective effects were observed in vivo in the natural settings of oxygen, nutrients, growth factors, and loading that occur within a knee joint. This suggests that our in vitro observations of chondroprotection (by metabolic shifting) can be replicated in vivo. 
It is unlikely that we could replicate galactose-replacement in vivo. However, that was not the goal of this study. Rather, this study demonstrated that reducing the pro-catabolic phenotype of OA may be obtained by targeting the root cause; namely fixing mitochondrial dysfunction, and that this may be a better approach than blocking cytokines or inhibiting MMPs. Even with control human OA chondrocytes, undetectable levels of pAMPK could be recovered by galactose-replacement, leading to inhibition of MMP13 protein production downstream. Thus, finding more promising pharmaceutical paths to generate mitochondrial reactivation in articular joint chondrocytes may lead to more effective therapeutic strategies to treat OA (or at least reduce OA progression) if used early enough in the disease. And it may not take full recovery of all mitochondria. Galactose-replacement rescued mitochondrial function better than 2DG (and 2DG matches HAS2-OE, 4MU, and DCA). However, galactose-replacement blocked increased MMP13 and cartilage breakdown to a similar extent as 2DG. Thus, forcing the activation of mitochondria, even to a small extent (as in 2DG), is sufficient for this rescue of the pro-catabolic phenotype.

\section{Methods}

Materials. Ham's F-12 and DMEM were obtained from Mediatech. Gibco ${ }^{\text {TM }}$ DMEM, no glucose, was obtained from Thermo Fisher scientific (11966025). D-(+)-Galactose was obtained from Sigma-Aldrich (G0750). All culture medium includes 50 units $/ \mathrm{ml}$ of Penicillin, $50 \mu \mathrm{g} / \mathrm{ml}$ of Streptomycin, $2 \mathrm{mM}$ of Lglutamate, and $25 \mu \mathrm{g} / \mathrm{ml}$ of Ascorbic acid. Galactose medium means Gibco ${ }^{\mathrm{TM}}$ DMEM, no glucose mixed with $10 \mathrm{mM}$ of Galactose, and Glucose medium means Ham's F-12 and DMEM as control. Oligomycin A was obtained from MP Biomedicals. FCCP, Rotenone, 2-deoxyglucose (2-DG), and Antimycin A were obtained from Sigma-Aldrich. Pronase (53702; EMD Millipore Calbiochem), collagenase P (11249002001; Roche Applied Science), were used in the dissociation of tissues. Fetal bovine serum (FBS) was from Hyclone. IL1 $\beta$ was obtained from R\&D Systems, Inc. Cell lysis buffer was obtained from Cell Signaling Technologies, and Clear Blue X-ray film was from Genesee Scientific. All other reagents were from Sigma-Aldrich.

Cell culture. Primary bovine articular chondrocytes were isolated from the articular cartilage of metacarpophalangeal joints of young adult steers (aged 18-24 months), which were obtained from a local slaughterhouse with approval from the North Carolina Department of Agriculture in the U.S.A. and Nagoya City Central Wholesale Market in Japan with institutional approval. No live animals were used in this study. Primary human articular chondrocytes were isolated from knee cartilage obtained from joint replacement surgery with institutional IRB approval (Nagoya University \#2020 - 0146). Document informed consent was obtained from all patients with the World Medical Association of Helsinki Ethical Principles for Medical Research Involving Human Subjects. Also, these tissues were obtained with no identifying information except age/sex. All methods were carried out in accordance with relevant guidelines and regulations. Human cartilage samples were from patients ( $75 \%$ female, $25 \%$ male) with an average age of $74 \pm 2.2$ years. Bovine and human chondrocytes were liberated from full-thickness slices of articular cartilage and cultured as described previously ${ }^{1,2}$. Chondrocytes were then incubated in a 
serum-free medium for $1 \mathrm{~h}$ prior to the addition of IL-1 $\beta(2 \mathrm{ng} / \mathrm{ml})$, and/or change to Galactose culture medium. Some experiments included co-treatment with 2-DG $(2 \mathrm{mM})$ as labeled. DMSO only at the same concentration was used as a control. Time courses varied depending on the experiment as labeled.

Cartilage explant cultures. Full-thickness $4 \mathrm{~mm}$ cores of bovine articular cartilage were cultured in $1.0 \mathrm{ml}$ of DMEM/Ham's F-12 medium containing $10 \%$ FBS for $48 \mathrm{~h}$. The medium was then replaced with serumfree Glucose and Galactose culture medium, and the tissues were incubated for 4-7 days in the presence of various activators, including IL-1 $\beta(3 \mathrm{ng} / \mathrm{ml})$ and 2DG. For histology, the treated explants were fixed with $4 \%$ buffered paraformaldehyde overnight at $4{ }^{\circ} \mathrm{C}$, rinsed in $30 \%$ sucrose, PBS, and embedded in paraffin. Sections $(8 \mu \mathrm{m})$ were prepared and stained with safranin $\mathrm{O}$ for the detection of proteoglycans and counterstained with Fast Green ${ }^{4}$. In other experiments, the culture medium aliquots were analyzed by a colorimetric assay for released proteoglycan content by a dimethyl methylene blue assay for s-GAG release ${ }^{4,29,30}$.

qRT-PCR. Total RNA was isolated from the bovine chondrocyte cultures as described previously ${ }^{1,2}$. Specific primers for real-time RT-PCR were custom-made by Integrated DNA Technologies (Coralville, IA). $\mathrm{RT}^{2}$ Real-Time ${ }^{\mathrm{TM}}$ SYBR Green reagents were from SA Biosciences. The bovine-specific primer sequences are as follows: MMP13 ${ }^{2}$, forward (5'-CCT GCT GGA ATC CTG AAG AAA-3') and reverse (5'-AGT CTG CCA GTC ACC TCT AA-3'); 18 s RNA ${ }^{2}$, forward (5'-GTA ACC CGT TGA ACC CCA TT-3') and reverse (5'-CCA TCC AAT CGG TAG TAG CG-3'); i-NOS, forward (5'-TAC CGC ACC CGA GAT GGC-3') and reverse (5'-TGG CAC TTC GCA CAA AGC A-3'). Real-time RT-PCR efficiency (E) was calculated as E $=10^{\left(-1 / \text { slope }^{\prime}\right)}{ }^{31}$. The-fold increase in copy numbers of mRNA was calculated as a relative ratio of a target gene to $18 \mathrm{~s}$ rRNA ( $\triangle \Delta$ $\mathrm{Ct}$ ), following the mathematical model introduced by Pfaff ${ }^{31}$, as described previously ${ }^{1,4}$.

Western blot analysis. Equivalent concentrations of protein lysates were loaded into 4-12\% NuPAGE Novex Tris-acetate gradient mini-gels (Thermo Fisher Scientific), electrophoresed, transferred to nitrocellulose, blocked and incubated with primary and secondary antibodies as described previously ${ }^{1,2}$. Band intensities were captured with a digital image scanner and quantified using densitometry software (C.S. Analyzer 3.0; ATTO, Tokyo, Japan) ${ }^{32}$. In some cases, the blots were stripped using Restore Plus Western Stripping Buffer (Thermo Fisher Scientific) for $30 \mathrm{~min}$ at room temperature and re-probed using another primary antibody. Specific antibodies used for analysis included rabbit polyclonal anti-MMP13 (18165-1-AP, lot 00010468, Proteintech), rabbit polyclonal anti-phospho-AMPKa ${ }^{2}$ (T172) (2535S, lot 10, Cell Signaling Technology), anti-total-AMPKa ${ }^{2}$ (D5A2) (5831S, lot 6, Cell Signaling Technology), antiPGC1 alpha (ab54481, lot GR3315850-1, Abcam), anti-beta-actin (4970S, lot 12, Cell Signaling Technology). Anti-rabbit IgG, HRP-linked Antibody (7074S, lot 25, Cell Signaling Technology).

Metabolomic studies using Seahorse flux analyzer. Bovine chondrocytes were plated at $8.0 \times 10^{4}$ cells/well into specially designed 96-well Seahorse XF cell culture microplates. The confluent monolayers were pre-incubated for $24 \mathrm{~h}$ with or without $2 \mathrm{ng} / \mathrm{ml} \mathrm{IL1} \beta$ and with or without the same level of $2 \mathrm{mM} 2-$ DG in Glucose or Galactose culture medium. The medium was changed to serum-free Seahorse XF Base 
Medium (without phenol red but with $10 \mathrm{mM}$ glucose, $1.0 \mathrm{mM}$ pyruvate, and $2.0 \mathrm{mM}$ glutamine added) or Seahorse XF DMEM, pH7.4, cells, depending on the assay. XF Mito stress test, XF glycolytic rate assay, XF Real time ATP rate assay were performed by following product guidelines as described previously ${ }^{1,2}$. Algorithms provided in Agilent assay report generator Excel files were used to generate plots and bar graphs.

Mitochondria staining. MitoPT TMRM Assay (TMRM) (Catalog\#: 9105, Immunochemistry Technologies) (10 $\mu \mathrm{M}$ final) and Mito Tracker ${ }^{\mathrm{TM}}$ Green FM (Mito Green) (Catalog\#: M7514, Thermo Fisher Scientific) (200 nM final) were used following the product information sheet. TMRM, which is a mitochondrial dye reagent having a delocalized positive charge, accumulates in an active, negatively charged mitochondrial membrane, and stains healthy mitochondria red. When the mitochondrial membrane potential collapses, TMRM becomes distributed throughout the cytosol. Mito Green stains all mitochondria green by reacting with the free thiol groups of cysteine residues belonging to mitochondrial proteins. Mitochondria were observed by a fluorescence microscope (BZ-X800, Keyence). The color intensity was quantified by an analysis software (Hybrid cell count, Keyence). Mito Green estimated the total number of all mitochondria; TMRM estimated the number of active mitochondria. TMRM / Mito Green Ratio, which is calculated by dividing the red color intensity of TMRM by the green color intensity of Mito Green is used for evaluation.

Nitric Oxide Assay. The culture media of some conditions were collected after 24 hours cell culture. The amount of Nitrite and Nitrate in the cell culture medium was measured by Greiss reagents ${ }^{32}$ : Nitric Oxide Assay Kit (Catalog \#: EMSNO, Invitrogen) following the product information sheet. The color change was quantified by absorbance, then the amounts calculated by using a standard curve. Nitric Oxide is considered as the sum of Nitrite and Nitrate in this study

Reactive Oxygen Species Assay. Intracellular Reactive Oxygen Species (ROS) were stained by the Cellular ROS Assay Kit (Catalog\#: ab113851, Abcam) following the product information sheet after 24 hours of cell culture. It includes the cell-permeant reagent; $2^{\prime}, 7^{\prime}$-dichlorofluoroscin diacetate (DCFDA), which stains intracellular ROS green. Stained-cells were observed by a fluorescence microscope (BZ-X800, Keyence), and the color intensity was quantified by an analysis software (Hybrid cell count, Keyence).

Statistical analysis. Values are expressed as mean \pm standard deviation (SD). One-way ANOVA followed by Tukey post-hoc tests are used for multiple-group comparisons and a two-tailed unpaired Student's ttest was used for direct comparison of the treatment group with control. A p-value of $<0.05$ was considered significant. $* p<0.05 ; * \star p<0.01$. Those statistics were performed by $E Z R^{33}$.

\section{Declarations}

\section{Acknowledgments}

The work was supported in part by a grant from the National Institutes of Health R21-AR072682 (WK) and, a grant from JSPS KAKENHI JP18K16678, and 19K09620 (KT and NT). The authors also thank Ms. 
Michelle Cobb and Ms. Joani Zary Oswald for their technical assistance as well as Dr. Jim Aloor, director of the Metabolomics Core Facility at East Carolina University.

\section{Author contributions}

Y.S., N.T., K.T., C.B.K., and W.K. contributed to the conception and design of the study, acquisition of data, analysis and interpretation of data, drafting/revising the manuscript critically for important intellectual content, and provided final approval of the version to be submitted. S.T., T.K. and S.I. contributed to the conception and design of the study, acquisition of data, revising the manuscript critically for important intellectual content, and provided final approval of the version to be submitted. W.K. and C.B.K. contributed to proofreading English. All authors read and approved the final manuscript.

\section{Competing interests}

Seikagaku corporation has provided research funding.

\section{Data availability}

All data generated or analyzed during this study are includes in this published article.

\section{References}

1. Ishizuka, S. et al. Hyaluronan synthase 2 (HAS2) overexpression diminishes the procatabolic activity of chondrocytes by a mechanism independent of extracellular hyaluronan. J Biol Chem 294, 1356213579, doi:10.1074/jbc.RA119.008567 (2019).

2. Terabe, K. et al. Chondroprotective effects of 4-methylumbelliferone and hyaluronan synthase-2 overexpression involve changes in chondrocyte energy metabolism. J Biol Chem 294, 17799-17817, doi:10.1074/jbc.RA119.009556 (2019).

3. Warburg, 0 . The Metabolism of Carcinoma Cells. The Journal of Cancer Research 9, 148-163, doi:10.1158/jcr.1925.148 (1925).

4. Ishizuka, S., Askew, E. B., Ishizuka, N., Knudson, C. B. \& Knudson, W. 4-Methylumbelliferone Diminishes Catabolically Activated Articular Chondrocytes and Cartilage Explants via a Mechanism Independent of Hyaluronan Inhibition. J Biol Chem 291, 12087-12104, doi:10.1074/jbc.M115.709683 (2016).

5. Frey, P. A. The Leloir pathway: a mechanistic imperative for three enzymes to change the stereochemical configuration of a single carbon in galactose. FASEB J 10, 461-470 (1996).

6. Aguer, C. et al. Galactose enhances oxidative metabolism and reveals mitochondrial dysfunction in human primary muscle cells. PLoS One 6, e28536, doi:10.1371/journal.pone.0028536 (2011).

7. Reitzer, L. J., Wice, B. M. \& Kennell, D. Evidence that glutamine, not sugar, is the major energy source for cultured HeLa cells. J Biol Chem 254, 2669-2676 (1979). 
8. Robinson, B. H., Petrova-Benedict, R., Buncic, J. R. \& Wallace, D. C. Nonviability of cells with oxidative defects in galactose medium: a screening test for affected patient fibroblasts. Biochem Med Metab Biol 48, 122-126, doi:10.1016/0885-4505(92)90056-5 (1992).

9. Graham, N. A. et al. Glucose deprivation activates a metabolic and signaling amplification loop leading to cell death. Mol Syst Bio/ 8, 589, doi:10.1038/msb.2012.20 (2012).

10. Li, X. et al. Targeting mitochondrial reactive oxygen species as novel therapy for inflammatory diseases and cancers. J Hematol Oncol 6, 19, doi:10.1186/1756-8722-6-19 (2013).

11. Poderoso, J. J., Helfenberger, K. \& Poderoso, C. The effect of nitric oxide on mitochondrial respiration. Nitric Oxide 88, 61-72, doi:10.1016/j.niox.2019.04.005 (2019).

12. Kolpen, M. et al. Nitric oxide production by polymorphonuclear leucocytes in infected cystic fibrosis sputum consumes oxygen. Clin Exp Immuno/ 177, 310-319, doi:10.1111/cei.12318 (2014).

13. Wang, M. et al. MMP13 is a critical target gene during the progression of osteoarthritis. Arthritis Res Ther 15, R5, doi:10.1186/ar4133 (2013).

14. Mobasheri, A. et al. The role of metabolism in the pathogenesis of osteoarthritis. Nat Rev Rheumatol 13, 302-311, doi:10.1038/nrrheum.2017.50 (2017).

15. Terkeltaub, R., Yang, B., Lotz, M. \& Liu-Bryan, R. Chondrocyte AMP-activated protein kinase activity suppresses matrix degradation responses to proinflammatory cytokines interleukin- $1 \beta$ and tumor necrosis factor a. Arthritis Rheum 63, 1928-1937, doi:10.1002/art.30333 (2011).

16. Liu-Bryan, R. Inflammation and intracellular metabolism: new targets in OA. Osteoarthritis Cartilage 23, 1835-1842, doi:10.1016/j.joca.2014.12.016 (2015).

17. Tchetina, E. V. \& Markova, G. A. Regulation of energy metabolism in the growth plate and osteoarthritic chondrocytes. Rheumatol Int 38, 1963-1974, doi:10.1007/s00296-018-4103-4 (2018).

18. June, R. K., Liu-Bryan, R., Long, F. \& Griffin, T. M. Emerging role of metabolic signaling in synovial joint remodeling and osteoarthritis. J Orthop Res 34, 2048-2058, doi:10.1002/jor.23420 (2016).

19. Marroquin, L. D., Hynes, J., Dykens, J. A., Jamieson, J. D. \& Will, Y. Circumventing the Crabtree effect: replacing media glucose with galactose increases susceptibility of HepG2 cells to mitochondrial toxicants. Toxico/ Sci 97, 539-547, doi:10.1093/toxsci/kfm052 (2007).

20. Diaz-Ruiz, R., Rigoulet, M. \& Devin, A. The Warburg and Crabtree effects: On the origin of cancer cell energy metabolism and of yeast glucose repression. Biochim Biophys Acta 1807, 568-576, doi:10.1016/j.bbabio.2010.08.010 (2011).

21. Leloir, L. F. Two decades of research on the biosynthesis of saccharides. Science 172, 1299-1303, doi:10.1126/science.172.3990.1299 (1971).

22. Grishko, V. et al. Effects of hyaluronic acid on mitochondrial function and mitochondria-driven apoptosis following oxidative stress in human chondrocytes. J Biol Chem 284, 9132-9139, doi:10.1074/jbc.M804178200 (2009).

23. Knudson, W., Ishizuka, S., Terabe, K., Askew, E. B. \& Knudson, C. B. The pericellular hyaluronan of articular chondrocytes. Matrix Bio/ 78-79, 32-46, doi:10.1016/j.matbio.2018.02.005 (2019). 
24. Rosenberg, J. H., Rai, V., Dilisio, M. F., Sekundiak, T. D. \& Agrawal, D. K. Increased expression of damage-associated molecular patterns (DAMPs) in osteoarthritis of human knee joint compared to hip joint. Mol Cell Biochem 436, 59-69, doi:10.1007/s11010-017-3078-x (2017).

25. Schaefer, L. Complexity of danger: the diverse nature of damage-associated molecular patterns. $J$ Biol Chem 289, 35237-35245, doi:10.1074/jbc.R114.619304 (2014).

26. Warburg, O., Geissler, A. W. \& Lorenz, S. [On growth of cancer cells in media in which glucose is replaced by galactose]. Hoppe Seylers Z Physiol Chem 348, 1686-1687 (1967).

27. Kase, E. T. et al. Remodeling of oxidative energy metabolism by galactose improves glucose handling and metabolic switching in human skeletal muscle cells. PLoS One 8, e59972, doi:10.1371/journal.pone.0059972 (2013).

28. Tsuchiya, S. et al. Suppression of murine osteoarthritis by 4-methylumbelliferone. J Orthop Res 38, 1122-1131, doi:10.1002/jor.24541 (2020).

29. Farndale, R. W., Sayers, C. A. \& Barrett, A. J. A direct spectrophotometric microassay for sulfated glycosaminoglycans in cartilage cultures. Connect Tissue Res 9, 247-248, doi:10.3109/03008208209160269 (1982).

30. Ono, Y., Ishizuka, S., Knudson, C. B. \& Knudson, W. Chondroprotective Effect of Kartogenin on CD44Mediated Functions in Articular Cartilage and Chondrocytes. Cartilage 5, 172-180, doi:10.1177/1947603514528354 (2014).

31. Rasmussen, T. B., Uttenthal, A., de Stricker, K., Belák, S. \& Storgaard, T. Development of a novel quantitative real-time RT-PCR assay for the simultaneous detection of all serotypes of foot-andmouth disease virus. Arch Viro/ 148, 2005-2021, doi:10.1007/s00705-003-0145-2 (2003).

32. Han, H. S., Qiao, Y., Karabiyikoglu, M., Giffard, R. G. \& Yenari, M. A. Influence of mild hypothermia on inducible nitric oxide synthase expression and reactive nitrogen production in experimental stroke and inflammation. J Neurosci 22, 3921-3928, doi:10.1523/jneurosci.22-10-03921.2002 (2002).

33. Kanda, Y. Investigation of the freely available easy-to-use software 'EZR' for medical statistics. Bone Marrow Transplant 48, 452-458, doi:10.1038/bmt.2012.244 (2013).

\section{Figures}



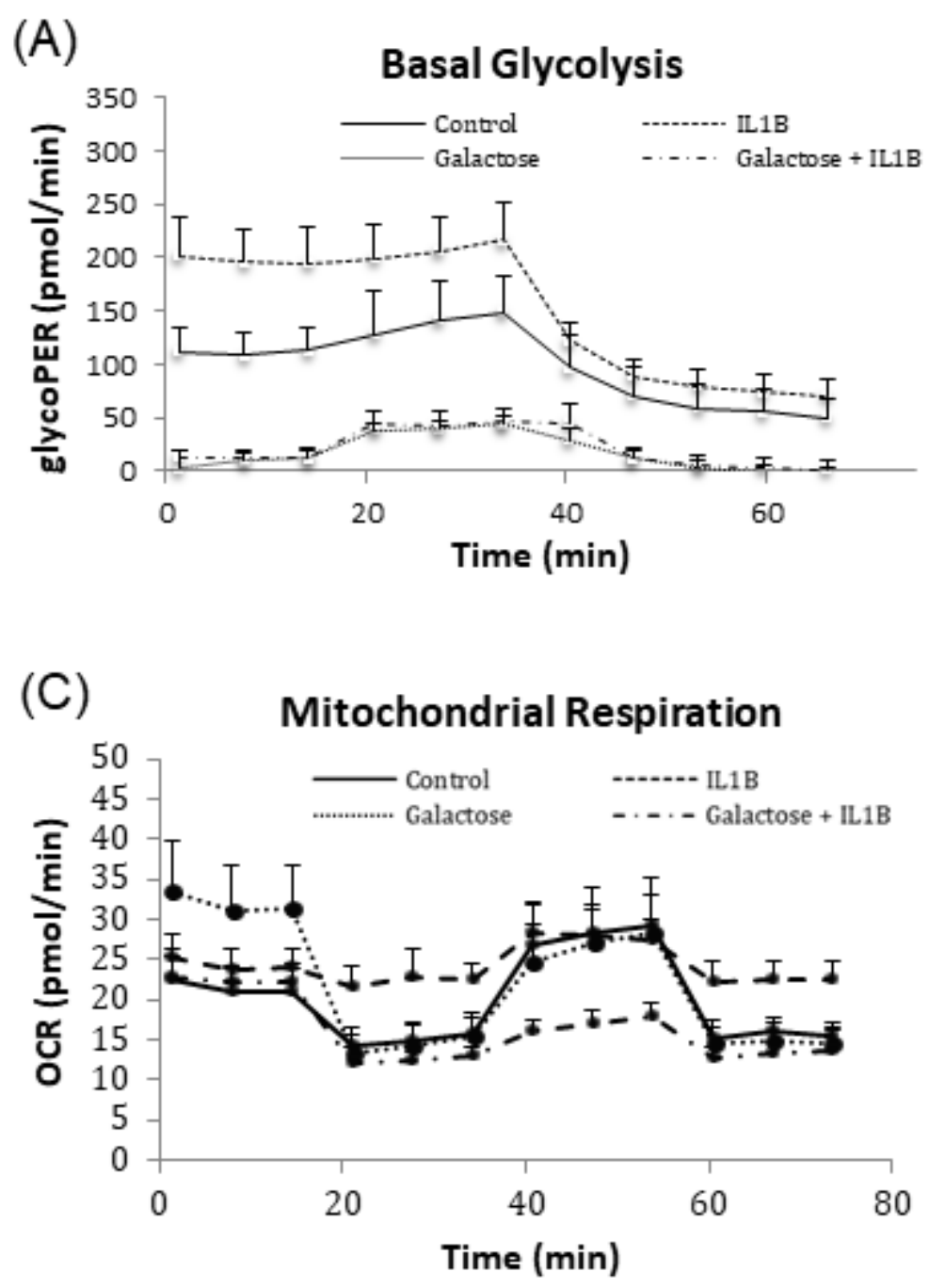

(B)

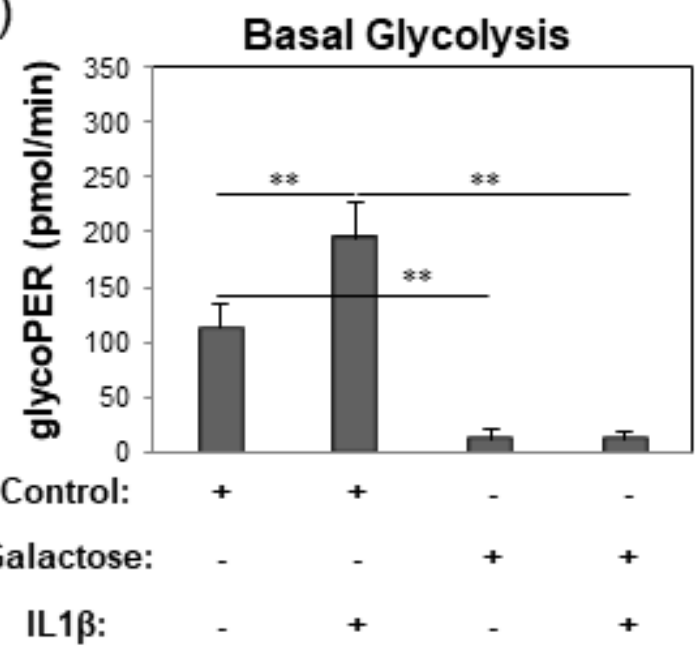

(D)

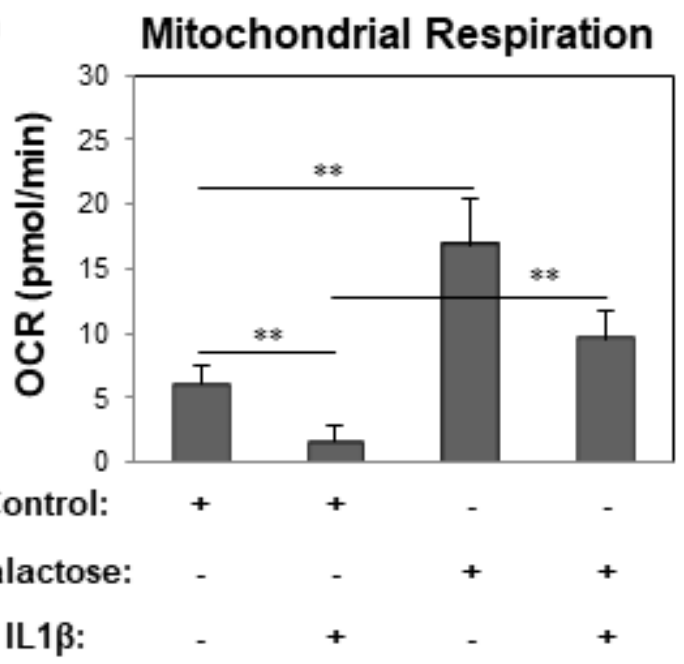

Figure 1

(A) Representative Glycolytic Rate Assay kinetic plot, wherein values for proton efflux rate (PER, $\mathrm{pmol} / \mathrm{min}$ ) are plotted versus time. Note that rotenone and antimycin A were injected at $18 \mathrm{~min}$ followed by high-dose 2DG injection at $36 \mathrm{~min}$. (B) Summaries of PER data representative of changes in basal glycolysis rates as labeled [mean \pm S.D. (error bars), $n=10$ ]. (C) Representative Mito Stress Test kinetic plot, wherein values for Oxygen Consumption Rate (OCR, $\mathrm{pmol} / \mathrm{min})$ are plotted versus time. Note that oligomycin A was injected at 18 min followed by FCCP injection at 36 min. rotenone and antimycin A were injected at 54min. (D) Summaries of OCR data representative of changes in basal mitochondrial respiration as labeled [mean \pm S.D. (error bars), $n=8$ ]. ANOVA followed by Tukey post-hoc test was used for statistical analysis. ${ }^{\star} p<0.05 ;{ }^{* \star} p<0.01$. 

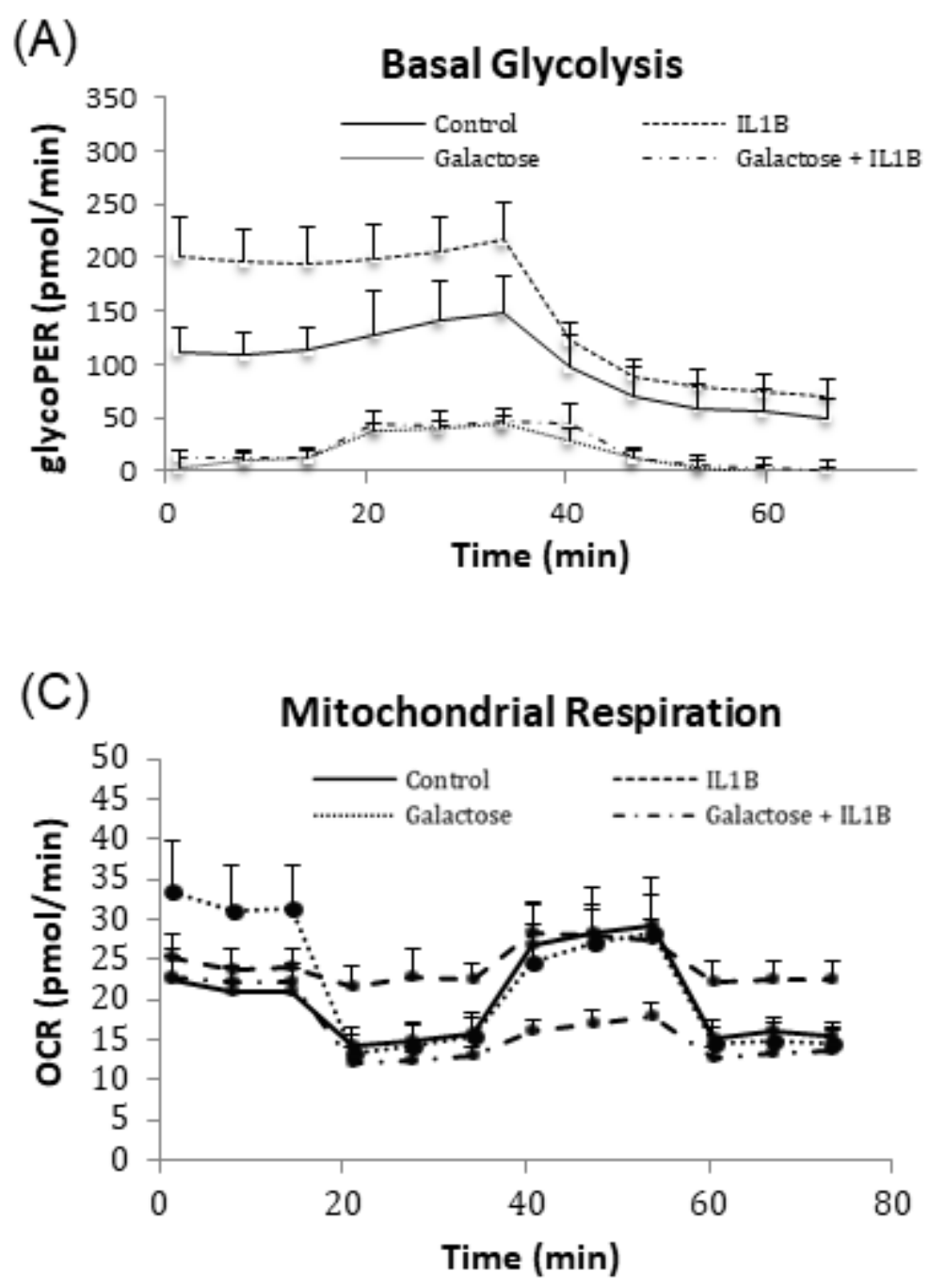

(B)

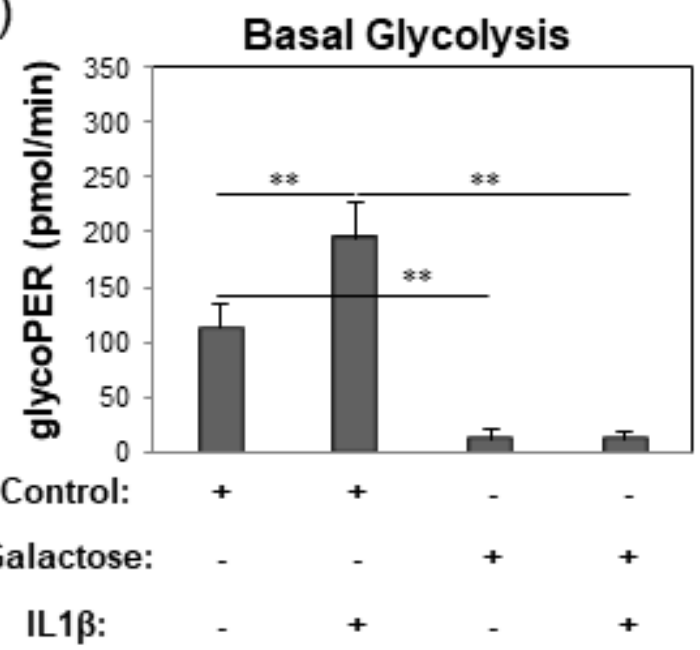

(D)

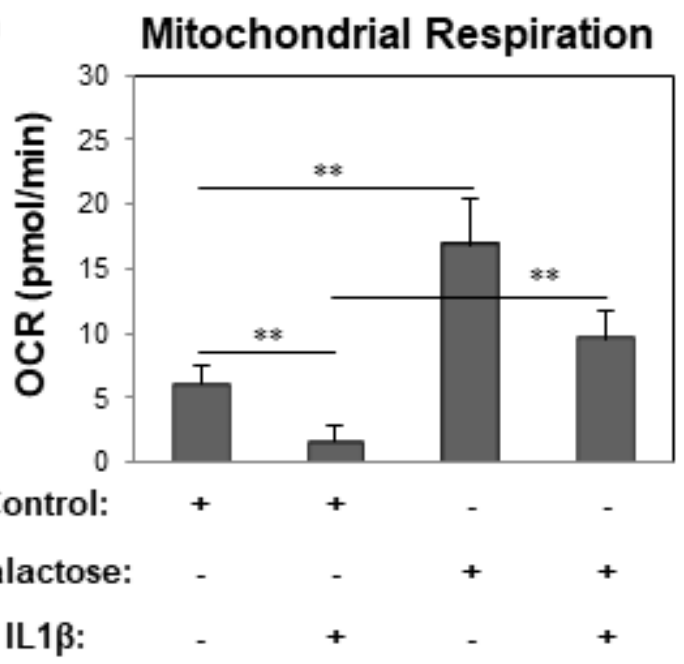

Figure 1

(A) Representative Glycolytic Rate Assay kinetic plot, wherein values for proton efflux rate (PER, $\mathrm{pmol} / \mathrm{min}$ ) are plotted versus time. Note that rotenone and antimycin A were injected at $18 \mathrm{~min}$ followed by high-dose 2DG injection at $36 \mathrm{~min}$. (B) Summaries of PER data representative of changes in basal glycolysis rates as labeled [mean \pm S.D. (error bars), $n=10$ ]. (C) Representative Mito Stress Test kinetic plot, wherein values for Oxygen Consumption Rate (OCR, $\mathrm{pmol} / \mathrm{min})$ are plotted versus time. Note that oligomycin A was injected at 18 min followed by FCCP injection at 36 min. rotenone and antimycin A were injected at 54min. (D) Summaries of OCR data representative of changes in basal mitochondrial respiration as labeled [mean \pm S.D. (error bars), $n=8$ ]. ANOVA followed by Tukey post-hoc test was used for statistical analysis. ${ }^{\star} p<0.05 ;{ }^{* \star} p<0.01$. 


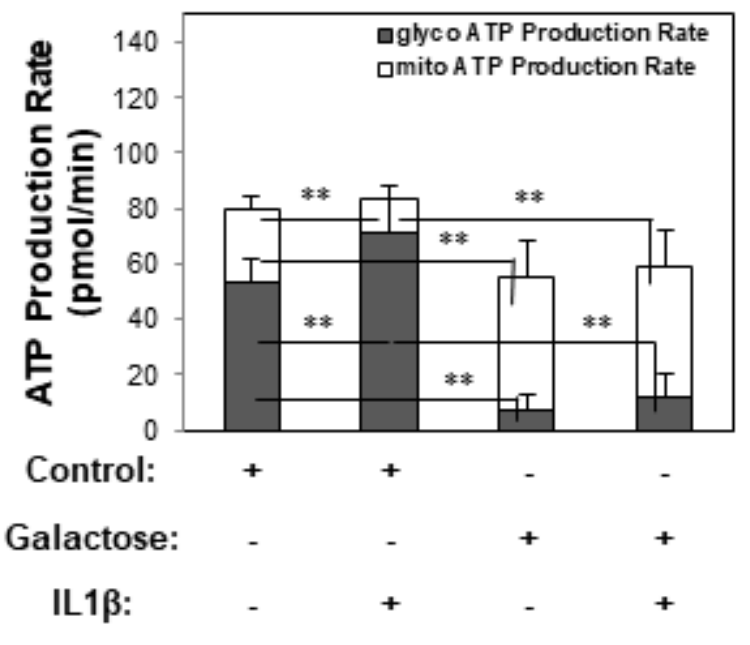

Figure 2

Galactose suppresses glycolysis and enhances mitochondrial respiration. Representative Real-Time ATP Production Rate Assay in bovine chondrocytes wherein the contribution of glycolysis (gray bars) and mitochondrial respiration (open bars) to ATP production after treatments for $24 \mathrm{hrs}$ are as labeled [mean \pm S.D. (error bars), $n=10$ ]. ANOVA followed by Tukey post-hoc test was used for statistical analysis. * $p$ $<0.05 ; * \star p<0.01$.

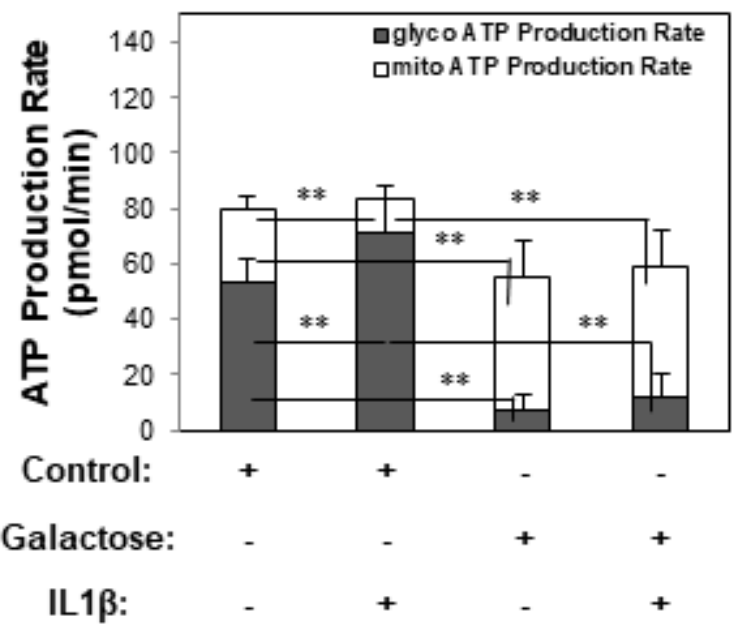

Figure 2

Galactose suppresses glycolysis and enhances mitochondrial respiration. Representative Real-Time ATP Production Rate Assay in bovine chondrocytes wherein the contribution of glycolysis (gray bars) and mitochondrial respiration (open bars) to ATP production after treatments for $24 \mathrm{hrs}$ are as labeled [mean \pm S.D. (error bars), $n=10$ ]. ANOVA followed by Tukey post-hoc test was used for statistical analysis. * $p$ $<0.05 ; * \star p<0.01$. 
(A)

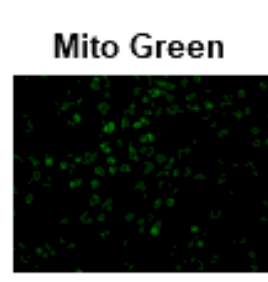

Control

FCCP

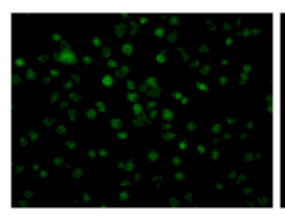

(C)
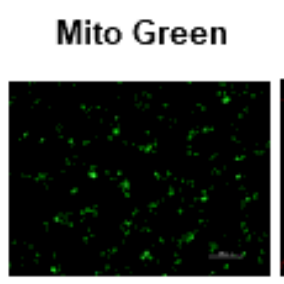

Control

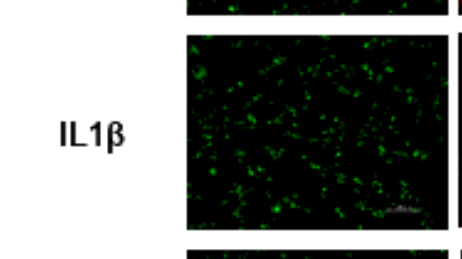

Galactose
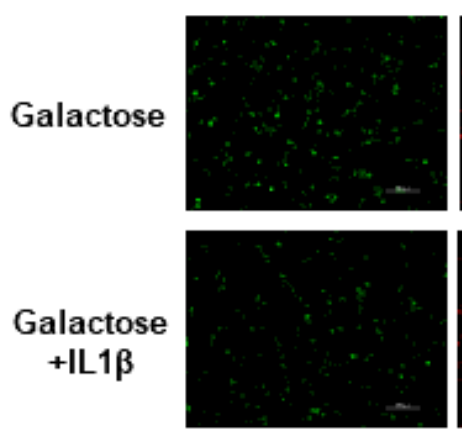

IL1 $\beta$

$+2-\mathrm{DG}$
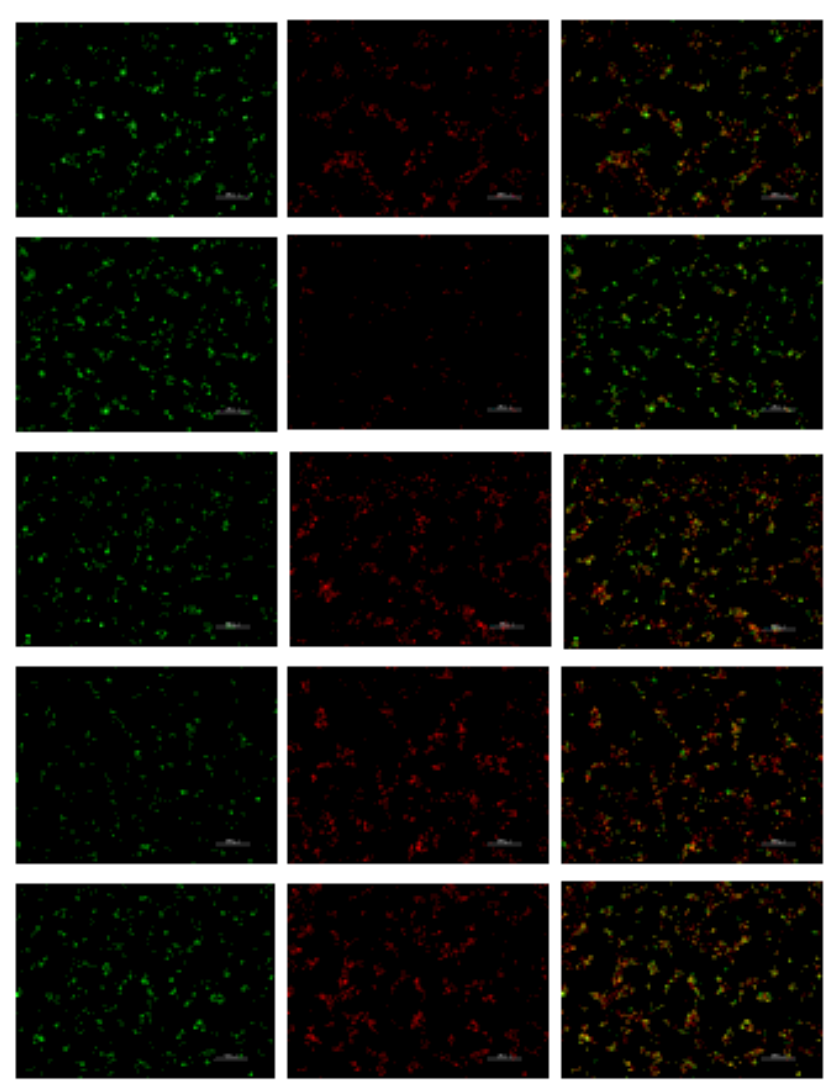

2-DG
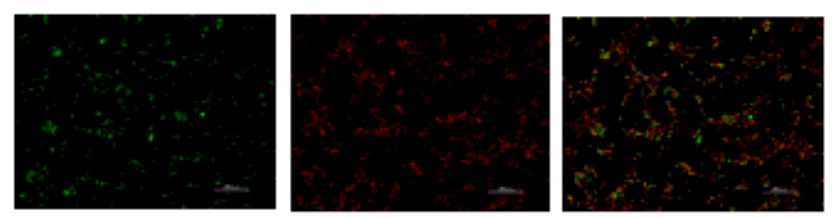

(B)

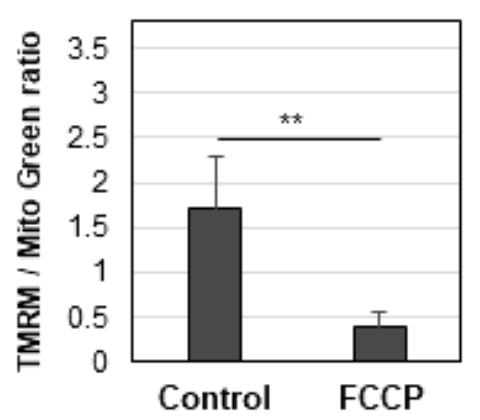

(D)

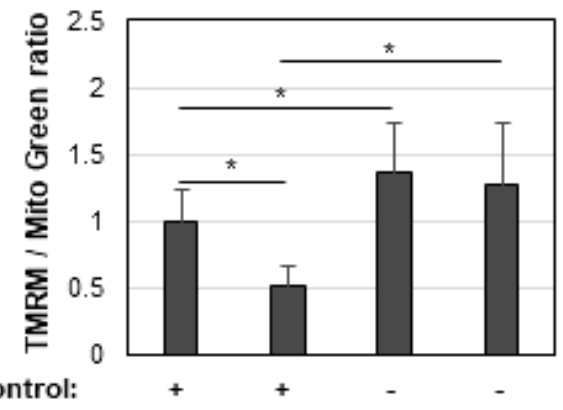

(E)

Galactose:

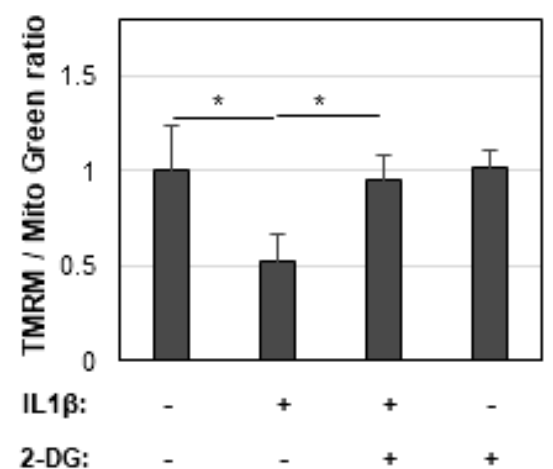

(F)

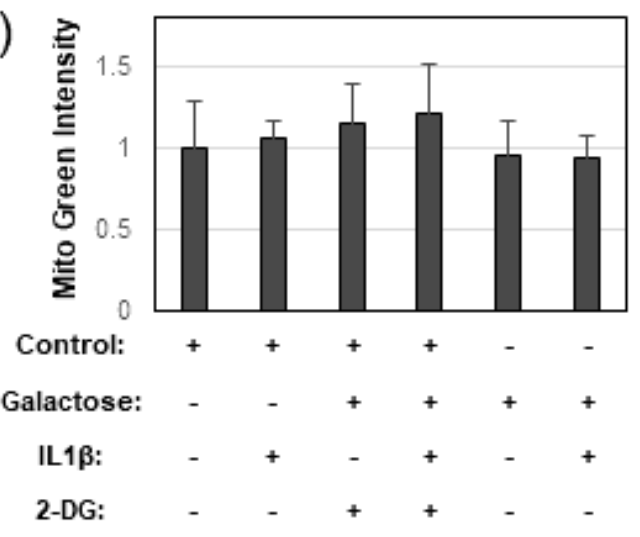

Figure 3

Effects of IL-1 $\beta$ and Galactose on mitochondrial integrity and function. (A) Representative images of Mito Green and TMRM stained chondrocytes in Control Glucose medium without or with FCCP. (B) Bar graph of TMRM / Mito Green ratio without or with FCCP. (C) Representative images of Mito Green, TMRM, and merge in Control Glucose or Galactose medium with or without IL1 $\beta$ and 2-DG as labeled. (D) Bar graph of TMRM / Mito Green ratio of Control, IL1 $\beta$, Galactose, Galactose+IL1 $\beta$ culture conditions. (E) Bar graph 
of TMRM / Mito Green ratio from Control Glucose, IL1 $\beta, I L 1 \beta+2 D G$, and 2DG culture conditions. (F) Bar graph of the color intensity of Mito Green alone in the conditions labeled. Displayed images ( $A$ and $C$ ) are digitally enhanced to $20 \%$ brighter. The color intensity (B, D, E, F) was calculated from raw images and bar graphs show the average \pm S.D. (error bars), $n=6$ experiments. Student's t-test was used for statistical analysis in panel B. ANOVA followed by Tukey post-hoc test was used for statistical analysis in panels D, $E$, and F. ${ }^{*} p<0.05 ; * * p<0.01$.

(A)

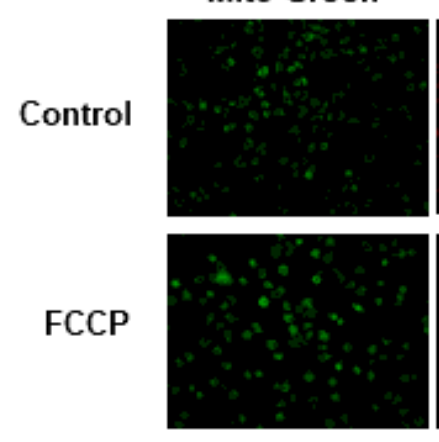

(C)

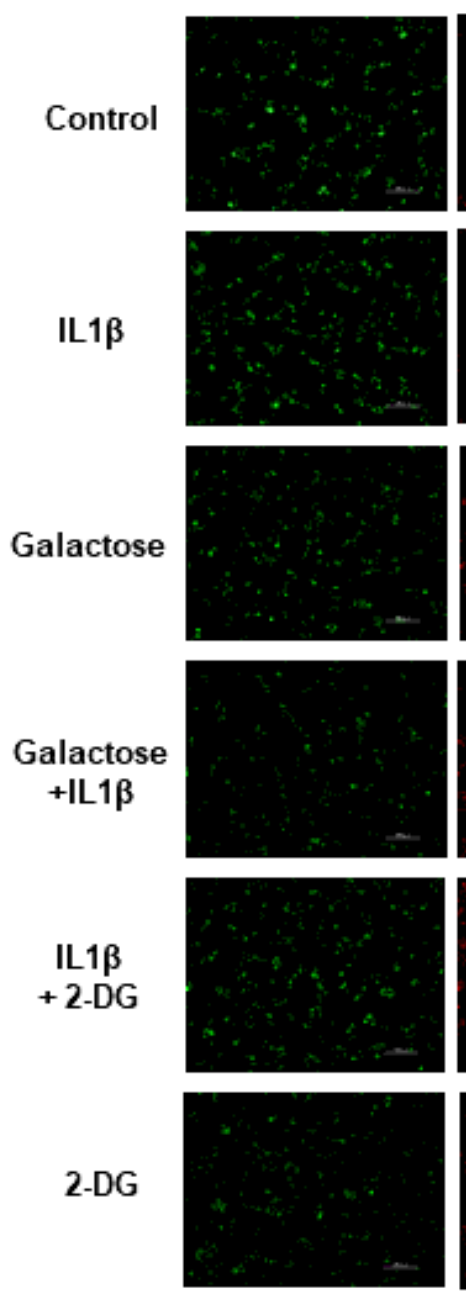

TMRM

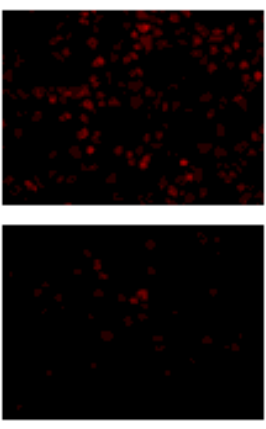

TMRM
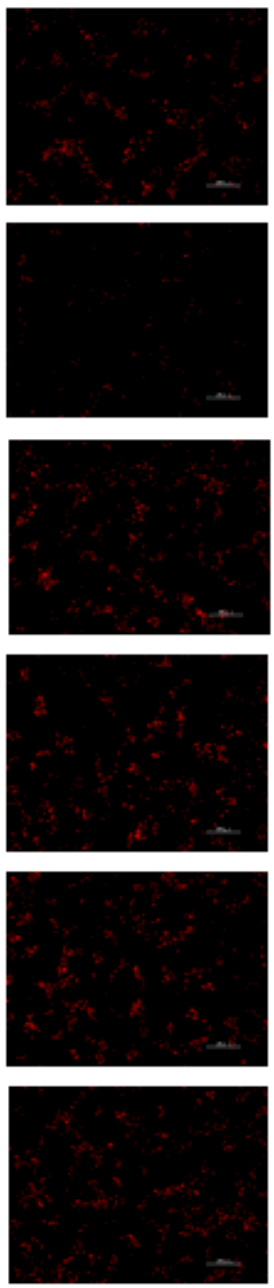

(B)

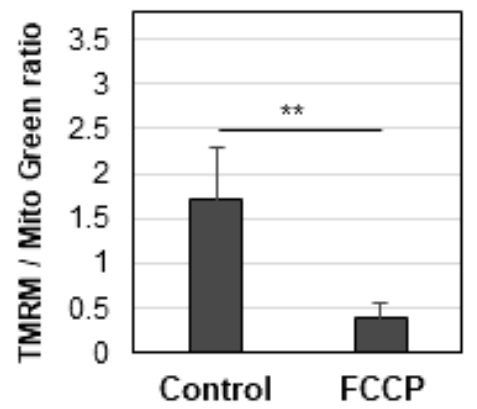

(D)
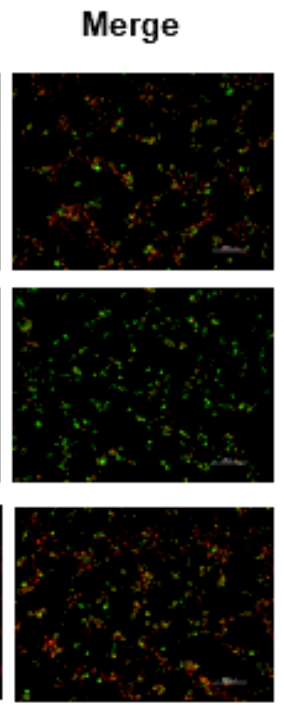

(E)

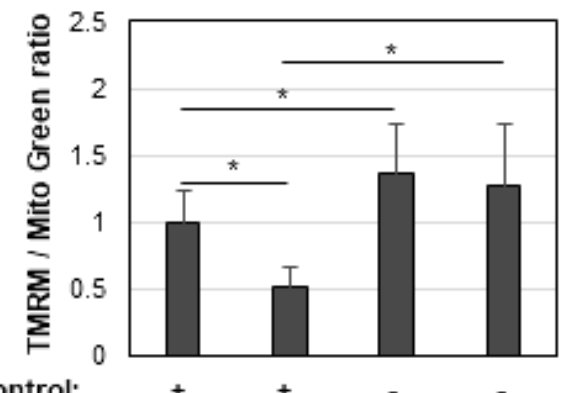

Galactose:

IL1 $\beta$ :

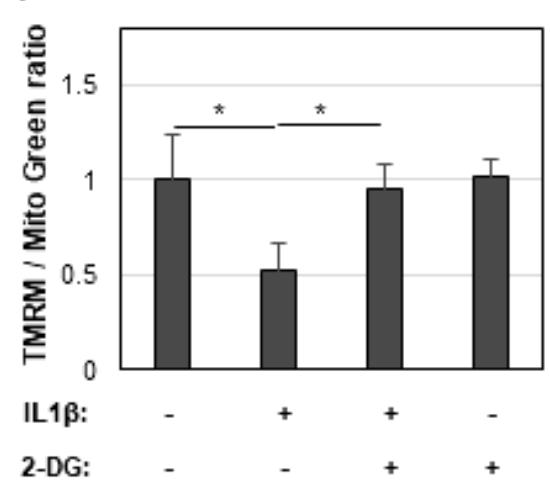

(F)
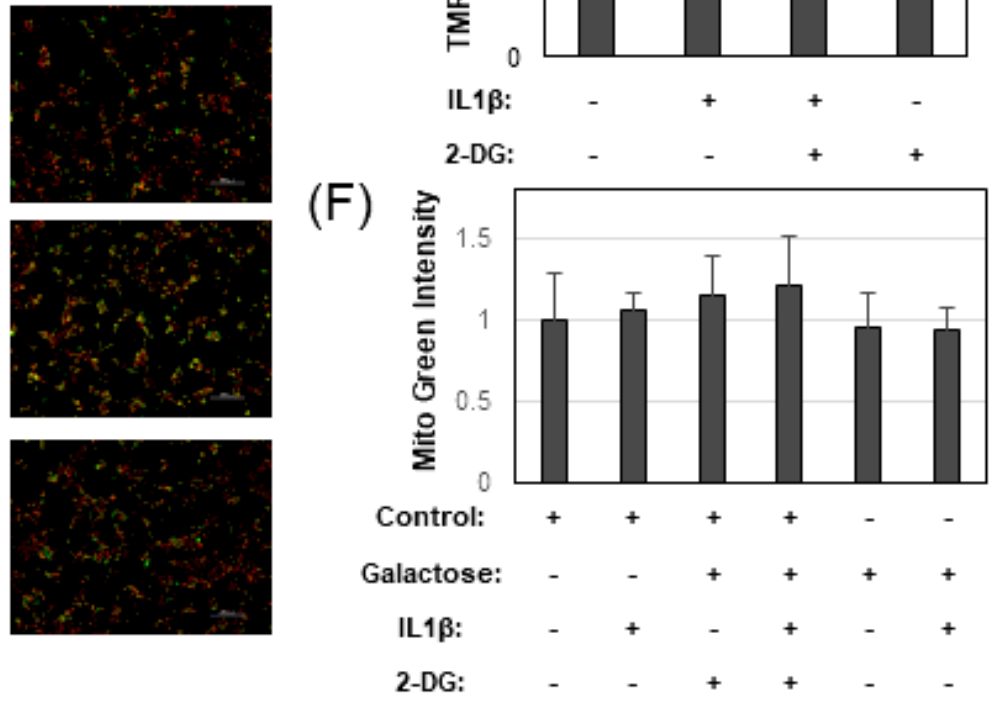
Effects of IL-1 $\beta$ and Galactose on mitochondrial integrity and function. (A) Representative images of Mito Green and TMRM stained chondrocytes in Control Glucose medium without or with FCCP. (B) Bar graph of TMRM / Mito Green ratio without or with FCCP. (C) Representative images of Mito Green, TMRM, and merge in Control Glucose or Galactose medium with or without IL1 $\beta$ and 2-DG as labeled. (D) Bar graph of TMRM / Mito Green ratio of Control, IL1 $\beta$, Galactose, Galactose+IL1 $1 \beta$ culture conditions. (E) Bar graph of TMRM / Mito Green ratio from Control Glucose, IL1 $\beta$, IL1 $\beta+2 D G$, and 2DG culture conditions. (F) Bar graph of the color intensity of Mito Green alone in the conditions labeled. Displayed images ( $A$ and $C$ ) are digitally enhanced to $20 \%$ brighter. The color intensity (B, D, E, F) was calculated from raw images and bar graphs show the average \pm S.D. (error bars), $n=6$ experiments. Student's t-test was used for statistical analysis in panel B. ANOVA followed by Tukey post-hoc test was used for statistical analysis in panels $D$, $\mathrm{E}$, and $\mathrm{F} .{ }^{*} \mathrm{p}<0.05 ; * * \mathrm{p}<0.01$.

(A)
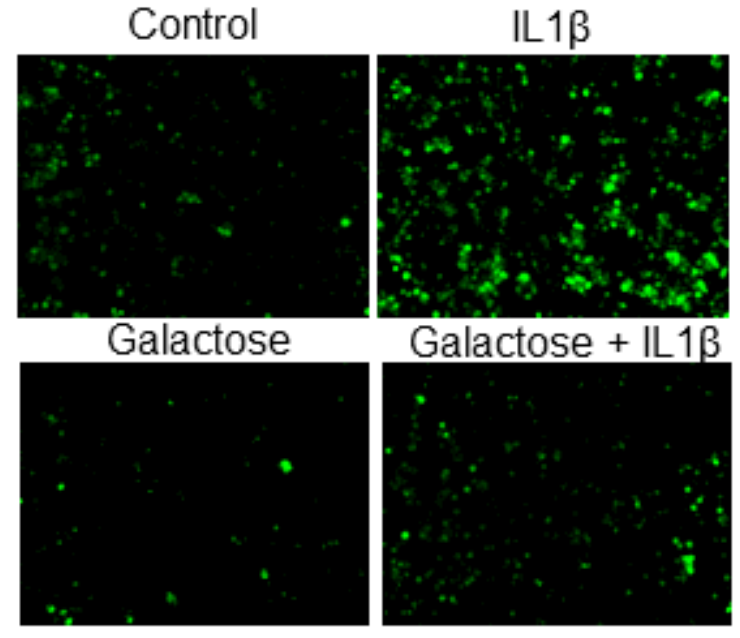

(C)

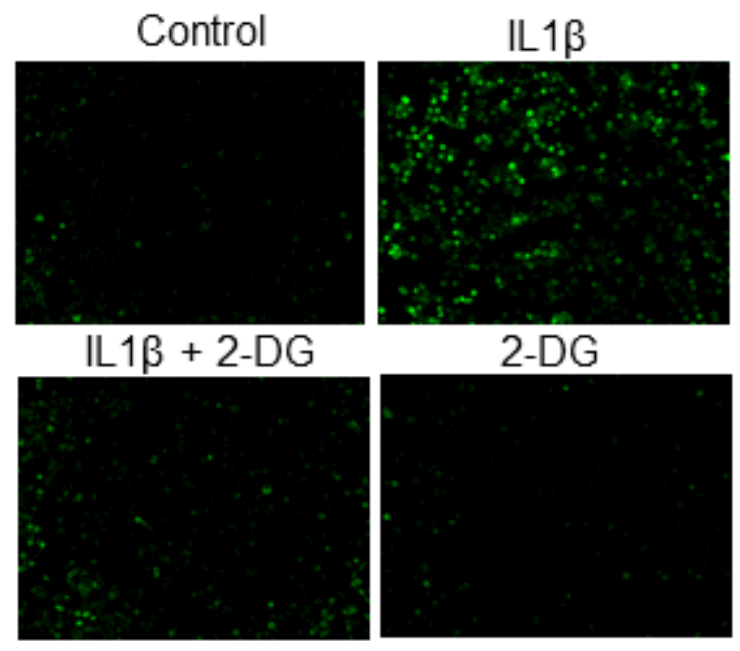

(B)

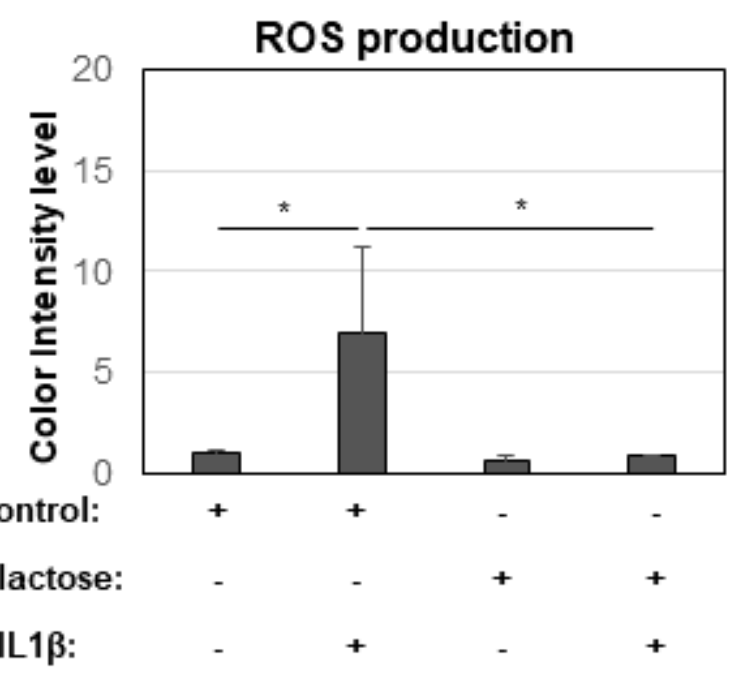

(D)

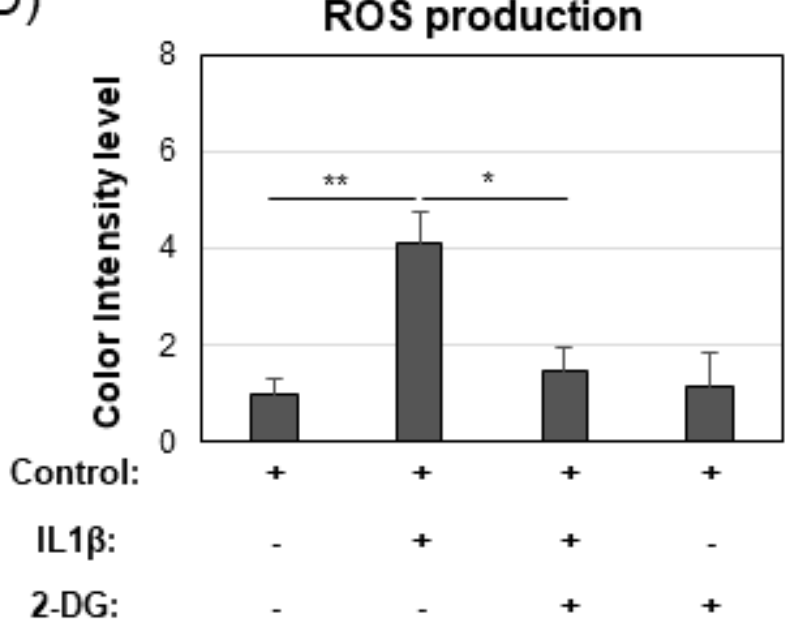

\section{Figure 4}

Galactose blocks ROS markers of mitochondrial damage. (A) Representative ROS Assay images for Control, IL1 $\beta$, Galactose, and Galactose+IL1 $\beta$ culture conditions. (B) The quantified color intensity from (A) [mean \pm S.D. (error bars), $n=3$ ]. (C) Representative ROS Assay images for Control, IL1 $\beta$, IL1 $\beta+2-D G$, 
and 2-DG. (D) The quantified color intensity from (C) [mean \pm S.D. (error bars), $n=3$ ]. ANOVA followed by Tukey post-hoc test was used for statistical analysis. ${ }^{*} p<0.05$; ${ }^{\star *} p<0.01$.

(A)
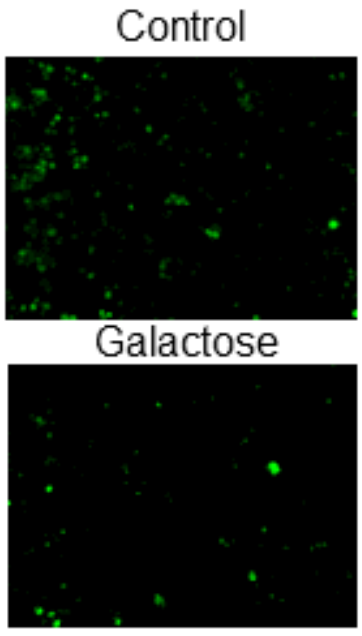

(C)

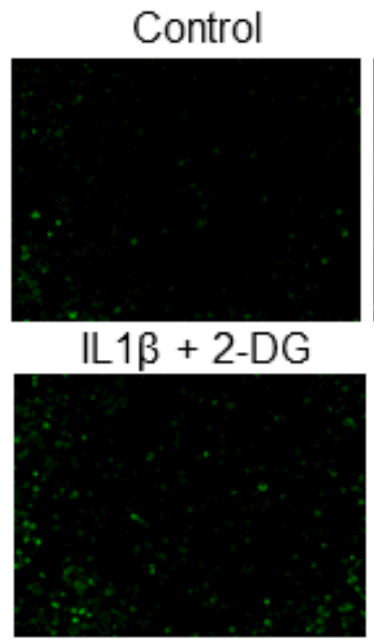

Galactose + IL $1 \beta$

IL $1 \beta$
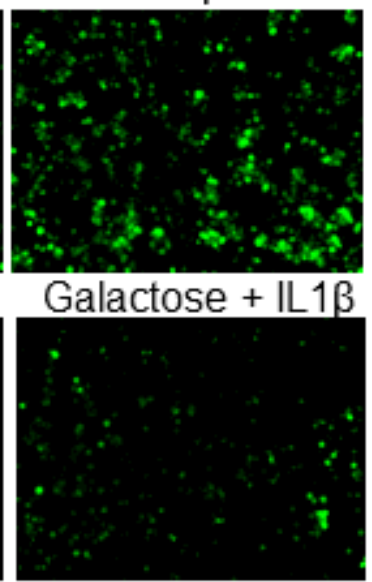

IL1 $\beta$

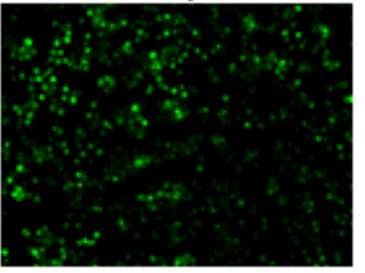

2-DG
(B)

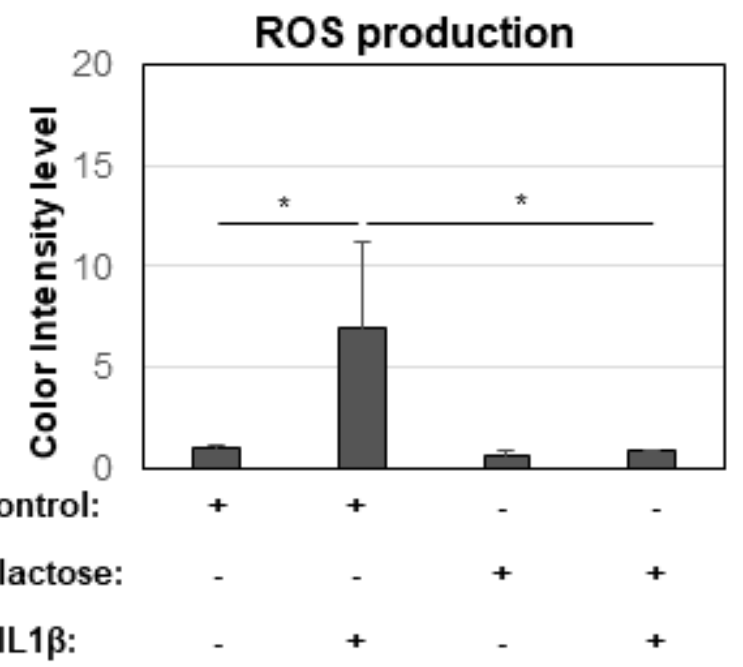

(D)

ROS production

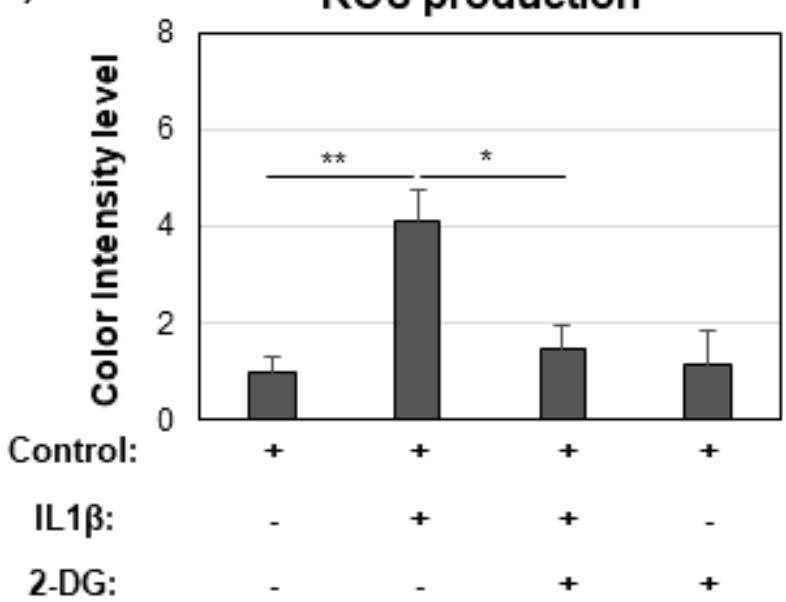

\section{Figure 4}

Galactose blocks ROS markers of mitochondrial damage. (A) Representative ROS Assay images for Control, IL $1 \beta$, Galactose, and Galactose+IL1 $\beta$ culture conditions. (B) The quantified color intensity from (A) [mean \pm S.D. (error bars), $n=3$ ]. (C) Representative ROS Assay images for Control, IL1 $\beta$, IL1 $\beta+2-D G$, and 2-DG. (D) The quantified color intensity from (C) [mean \pm S.D. (error bars), $n=3$ ]. ANOVA followed by Tukey post-hoc test was used for statistical analysis. ${ }^{\star} p<0.05 ;{ }^{\star \star} p<0.01$. 
(A)

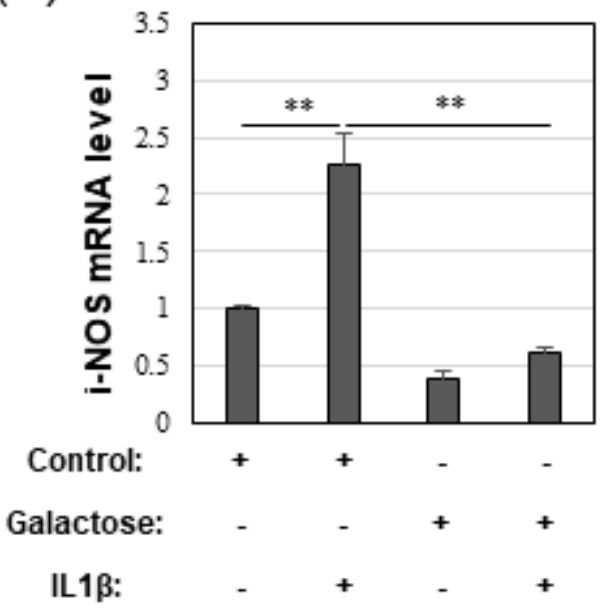

(B)

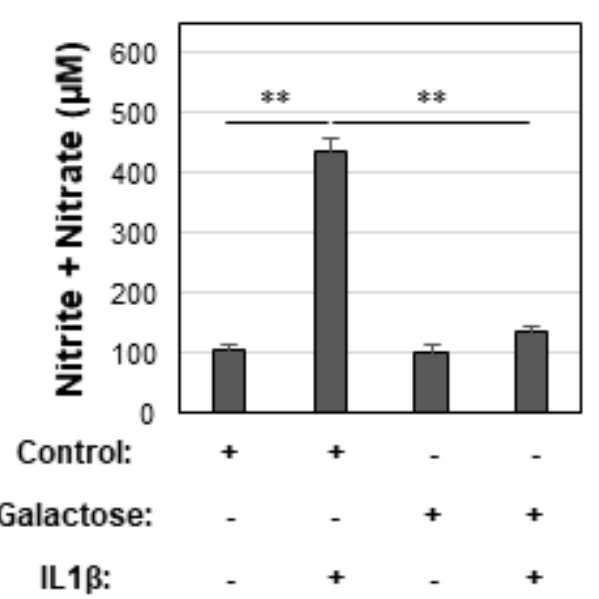

(C)

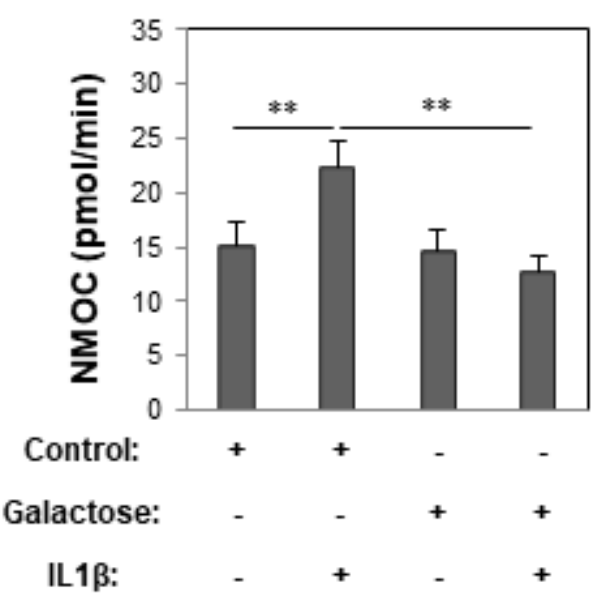

Figure 5

Galactose blocks NO and NMOC markers of mitochondrial damage. (A) The mRNA of inducible nitric oxide synthase (i-NOS) was measured by RT-Real Time PCR for chondrocytes in Control Glucose or Galactose culture condition treated with or without IL1 $\beta$ [mean \pm S.D. (error bars), $n=3$ ]. (B) The amount of Nitric Oxide in cell culture medium was determined for chondrocytes in Control Glucose or Galactose culture condition treated with or without IL $1 \beta$ [mean \pm S.D. (error bars), $n=3$ ]. (C) Non mitochondrial Oxygen Consumption Rate (OCR) by chondrocytes treated with or without IL1 $1 \beta$ in Control Glucose or Galactose cultures as labeled [mean \pm S.D. (error bars), $n=8$ ]. ANOVA followed by Tukey post-hoc test was used for statistical analysis. ${ }^{*} p<0.05 ;{ }^{*} \mathrm{p}<0.01$.

(A)

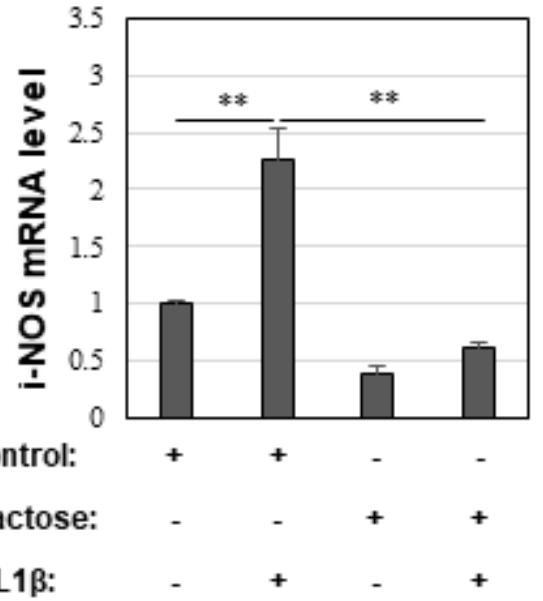

(B)

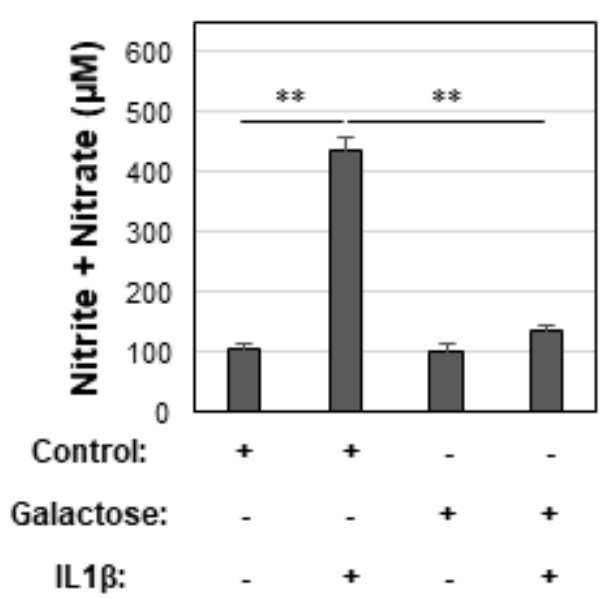

(C)

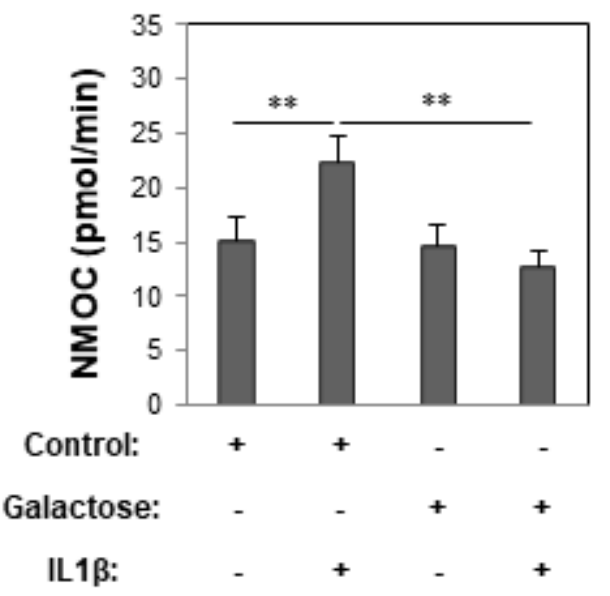

\section{Figure 5}

Galactose blocks NO and NMOC markers of mitochondrial damage. (A) The mRNA of inducible nitric oxide synthase (i-NOS) was measured by RT-Real Time PCR for chondrocytes in Control Glucose or Galactose culture condition treated with or without IL1 $\beta$ [mean \pm S.D. (error bars), $n=3$ ]. (B) The amount of Nitric Oxide in cell culture medium was determined for chondrocytes in Control Glucose or Galactose 
culture condition treated with or without IL $1 \beta$ [mean \pm S.D. (error bars), $n=3$ ]. (C) Non mitochondrial Oxygen Consumption Rate (OCR) by chondrocytes treated with or without IL1 $\beta$ in Control Glucose or Galactose cultures as labeled [mean \pm S.D. (error bars), $n=8$ ]. ANOVA followed by Tukey post-hoc test was used for statistical analysis. ${ }^{*} p<0.05 ;{ }^{* *} p<0.01$.

(A)

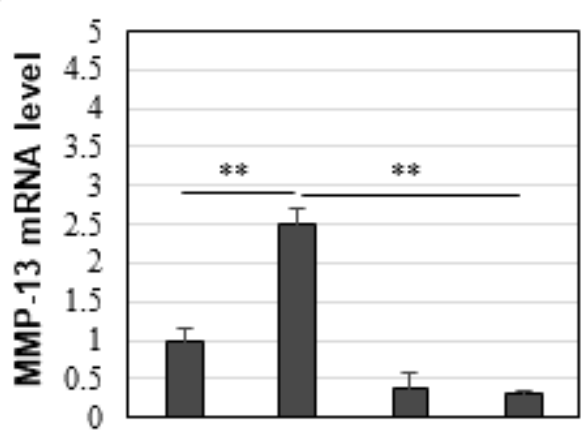

Control:

Galactose: - $\quad$ - $\quad+\quad+$

IL1ß:

(C)

$$
\text { Control }
$$

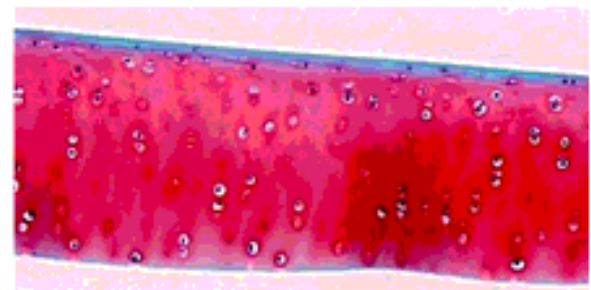

Galactose

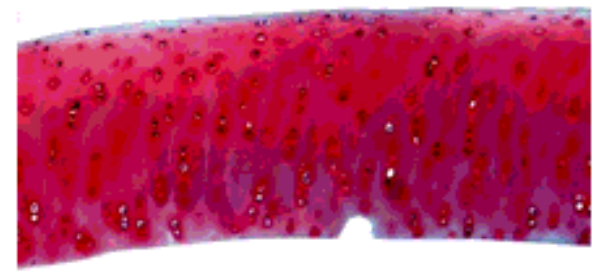

(B)

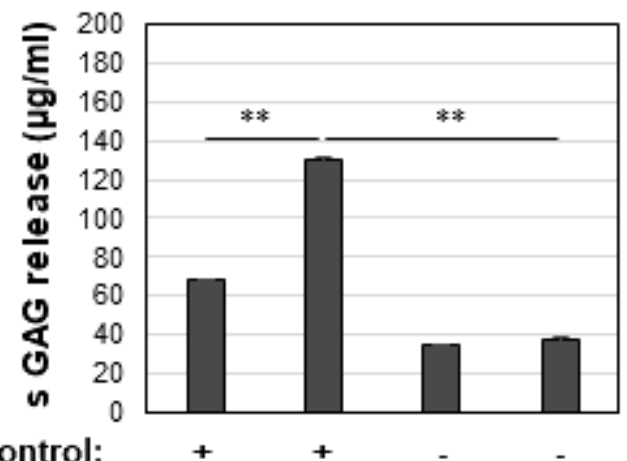

Galactose:

IL1 $\beta$ :

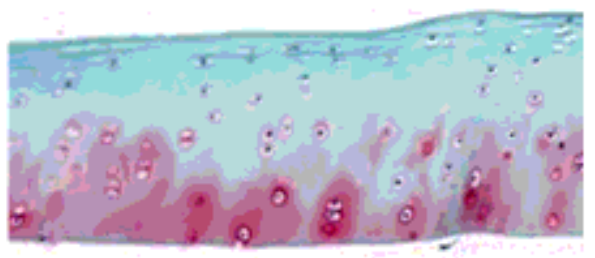

Galactose + IL1 $\beta$

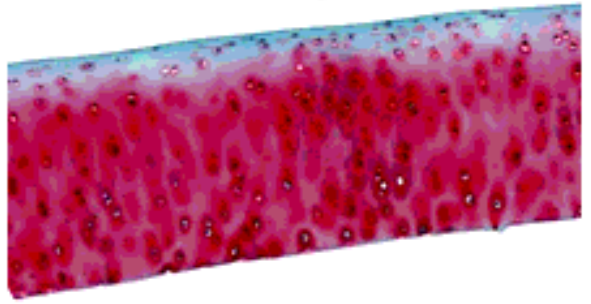

\section{Figure 6}

Galactose-replaced culture conditions block pro-catabolic features of activated chondrocytes and cartilage. (A) Expression of MMP-13 mRNA of by bovine chondrocytes treated with or without IL1 $\beta$ in Control Glucose or Galactose culture medium [untreated control value set $=1.0$; mean \pm S.D. (error bars), $n=3]$. (B) Quantified sulfated glycosaminoglycan released or lost from the tissue and accumulated in the medium by a colorimetric: Dimethyl-Methylene Blue (DMMB) assay (after 3 days) of bovine explant cultures [mean \pm S.D. (error bars), $n=4$ ]. (C) Representative images of bovine cartilage sections stained by Safranin $O$ and Fast Green from explant cultures treated with or without $3 \mathrm{ng} / \mathrm{ml}$ IL $1 \beta$ in Control 
Glucose or Galactose culture medium for 7 days $(n=4)$. ANOVA followed by Tukey post-hoc test was used for statistical analysis. ${ }^{*} p<0.05 ;{ }^{* *} p<0.01$.

(A)
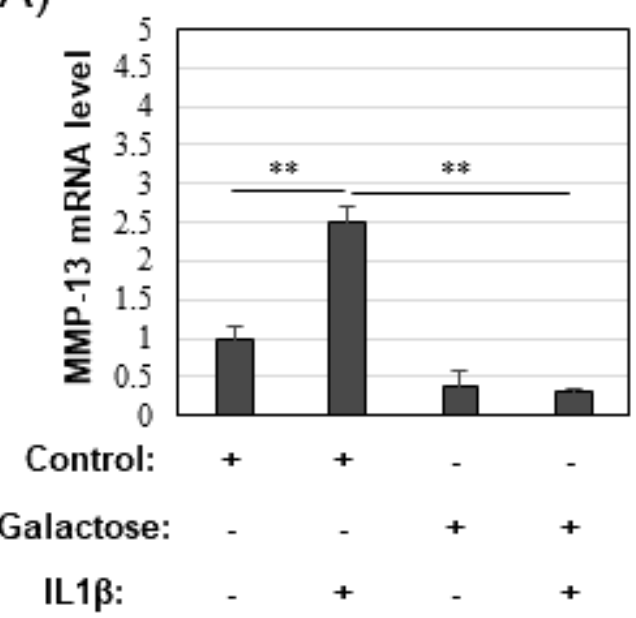

(C)

\section{Control}

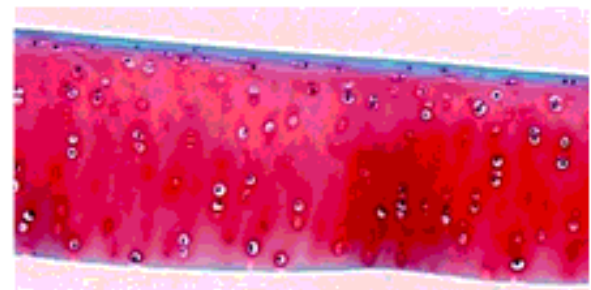

\section{Galactose}

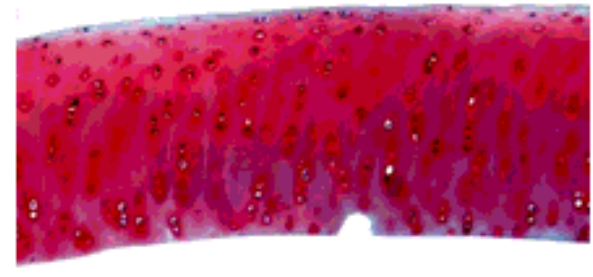

(B)

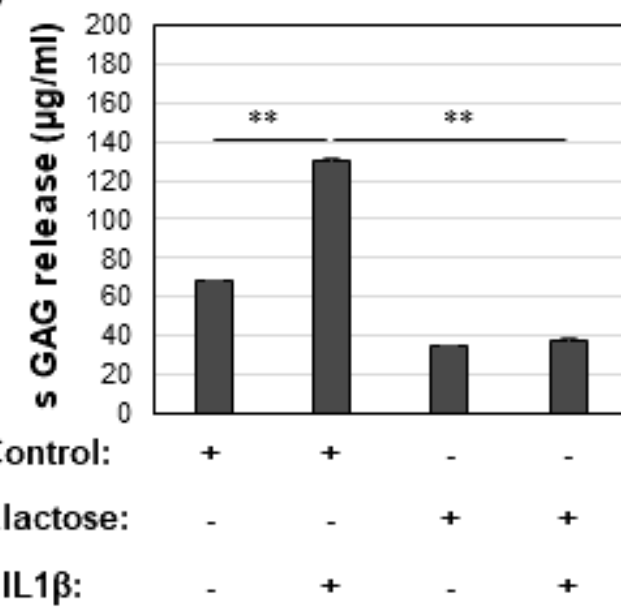

\section{IL1 $\beta$}

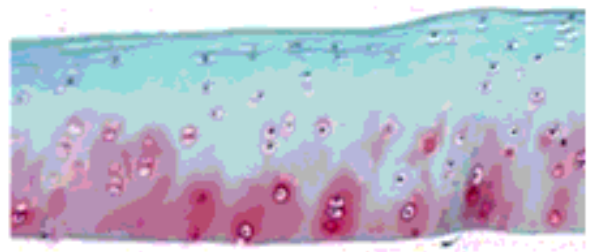

Galactose + IL1 $\beta$

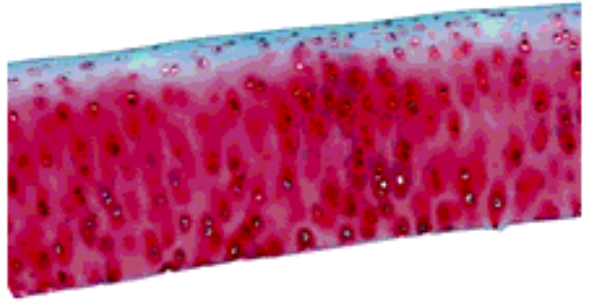

\section{Figure 6}

Galactose-replaced culture conditions block pro-catabolic features of activated chondrocytes and cartilage. (A) Expression of MMP-13 mRNA of by bovine chondrocytes treated with or without IL1 $\beta$ in Control Glucose or Galactose culture medium [untreated control value set $=1.0$; mean \pm S.D. (error bars), $n=3]$. (B) Quantified sulfated glycosaminoglycan released or lost from the tissue and accumulated in the medium by a colorimetric: Dimethyl-Methylene Blue (DMMB) assay (after 3 days) of bovine explant cultures [mean \pm S.D. (error bars), $n=4$ ]. (C) Representative images of bovine cartilage sections stained by Safranin $O$ and Fast Green from explant cultures treated with or without $3 \mathrm{ng} / \mathrm{ml} \mathrm{IL1 \beta}$ in Control Glucose or Galactose culture medium for 7 days $(n=4)$. ANOVA followed by Tukey post-hoc test was used for statistical analysis. ${ }^{\star} p<0.05 ;{ }^{* \star} p<0.01$. 

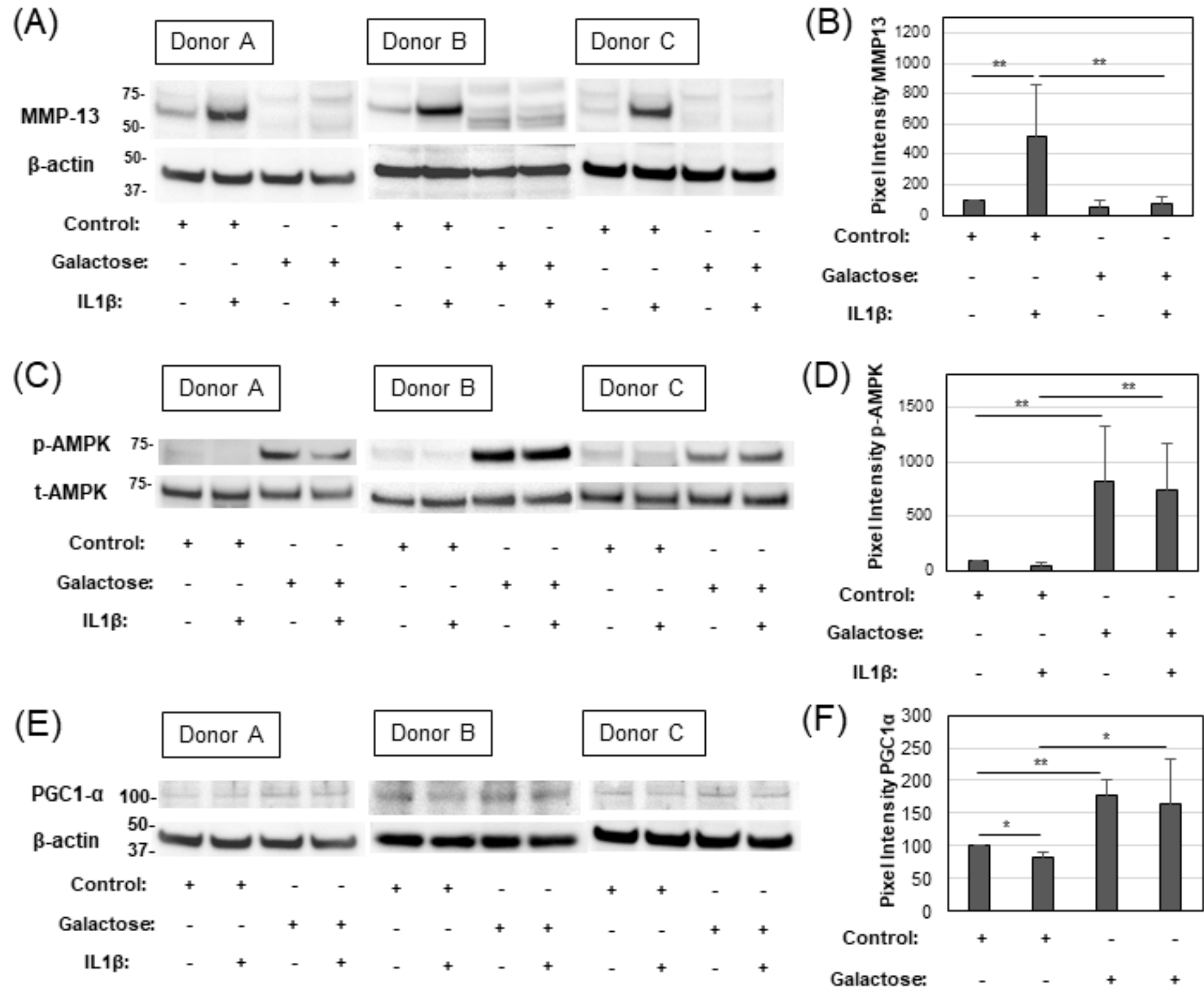

(F)

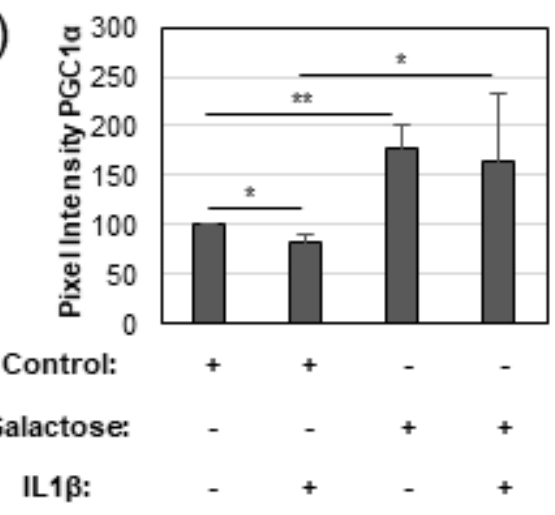

Figure 7

Effects of IL-1 $\beta$ and galactose on human OA chondrocytes. Human chondrocyte lysate from 3 donors were used in this study. Donor $A$ is a 77 years old woman, Donor $B$ is a 73 years old woman, Donor $C$ is a 77 years old woman. (A) Representative western blots of the expression of MMP-13 protein of Human chondrocytes (3 different donors) treated with or without IL1 $\beta$ in Control Glucose or Galactose culture medium. The grouping of gels/blots cropped from different parts of the same gel. Full-length gels and blots are included in a Supplementary Information file. (B) Densitometry of MMP13/ $\beta$-actin. Values are mean \pm standard deviation from three independent experiments and expressed as $n$-fold increase compared to untreated control set to 100. (C) Representative western blots of phospho-AMPK (p-AMPK) and total-AMPK (t-AMPK). (D) Densitometry of p-AMPK/AMPK. Values are mean \pm standard deviation from three independent experiments and expressed as $n$-fold increase compared to untreated control set to 100. (E) Representative western blots of PGC1a and $\beta$-actin. (F) Densitometry of PGC1 $\alpha / \beta$-actin. 
Values are mean \pm standard deviation from three independent experiments and expressed as $n$-fold increase compared to untreated control set to 100. ANOVA followed by Tukey post-hoc test was used for statistical analysis. ${ }^{\star} p<0.05 ;{ }^{* \star} p<0.01$. The full-length gels are seen in the Supplementary Information.
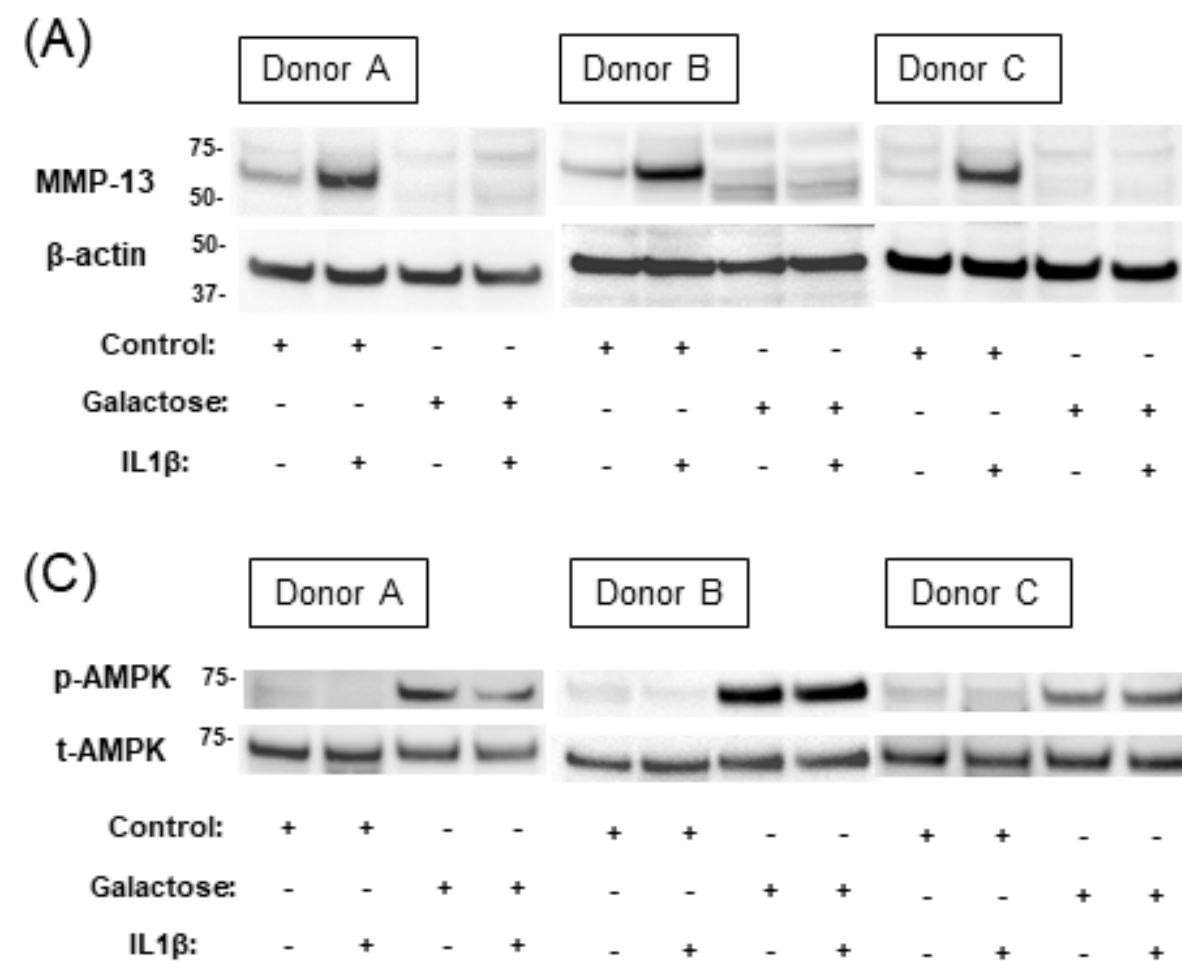

$\begin{array}{lllllll}- & - & + & + & - & + & +\end{array}$
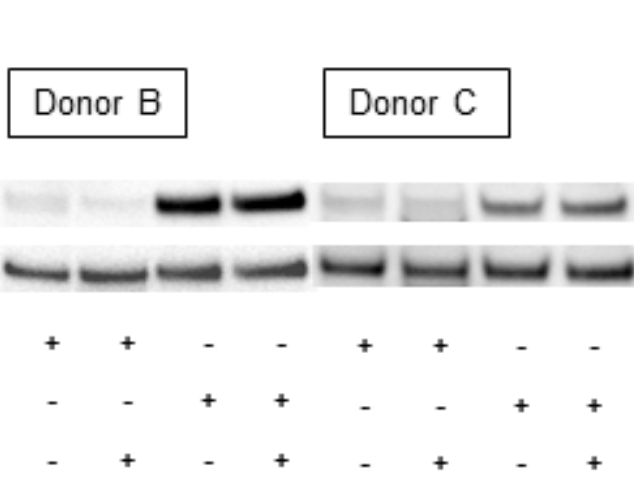

(E)
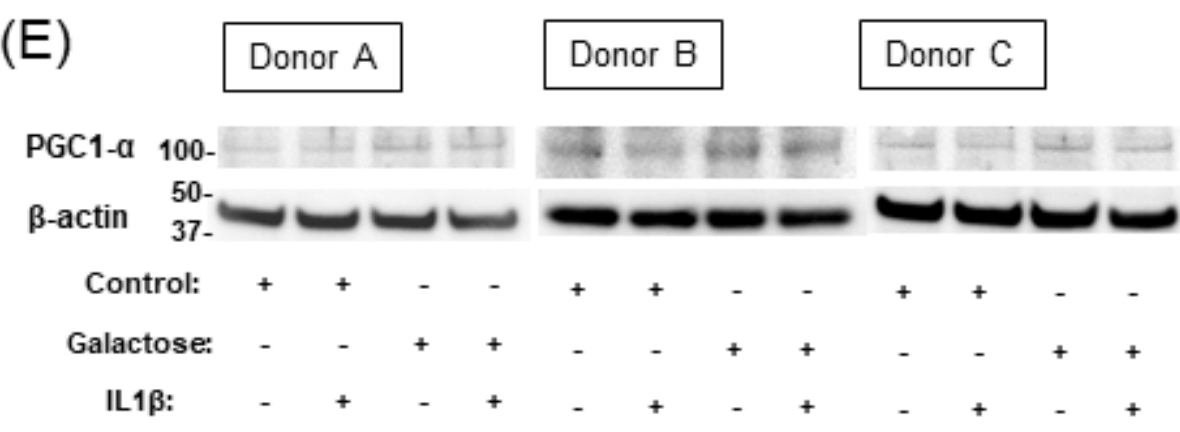

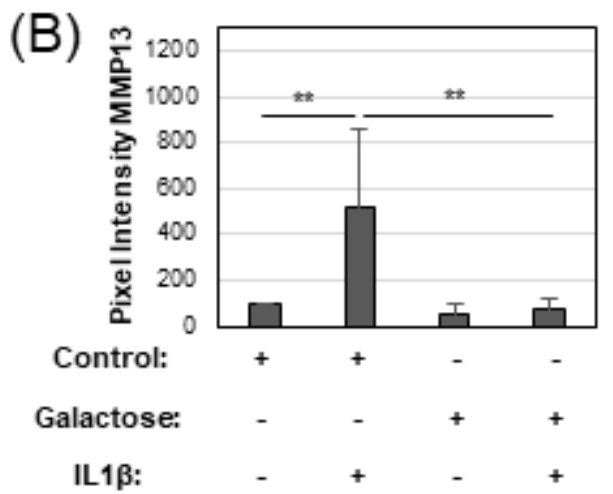

(D)

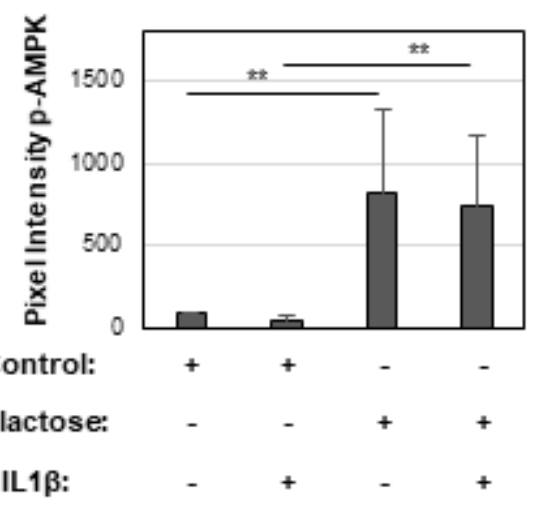

$(\mathrm{F})$

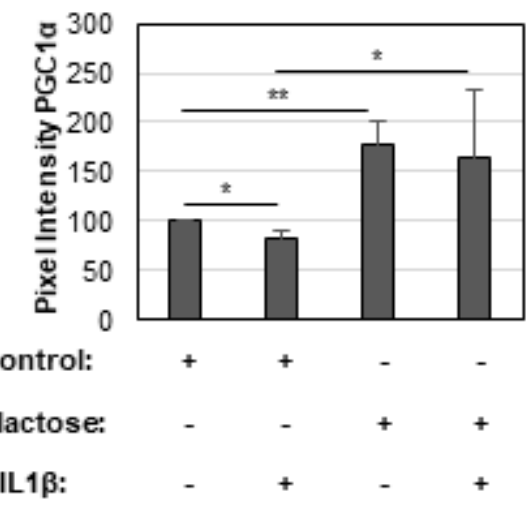

\section{Figure 7}

Effects of IL-1 $\beta$ and galactose on human OA chondrocytes. Human chondrocyte lysate from 3 donors were used in this study. Donor $A$ is a 77 years old woman, Donor $B$ is a 73 years old woman, Donor $C$ is a 77 years old woman. (A) Representative western blots of the expression of MMP-13 protein of Human chondrocytes (3 different donors) treated with or without IL1 $\beta$ in Control Glucose or Galactose culture medium. The grouping of gels/blots cropped from different parts of the same gel. Full-length gels and blots are included in a Supplementary Information file. (B) Densitometry of MMP13/ $\beta$-actin. Values are mean \pm standard deviation from three independent experiments and expressed as $n$-fold increase compared to untreated control set to 100. (C) Representative western blots of phospho-AMPK (p-AMPK) 
and total-AMPK (t-AMPK). (D) Densitometry of p-AMPK/AMPK. Values are mean \pm standard deviation from three independent experiments and expressed as $n$-fold increase compared to untreated control set to 100. (E) Representative western blots of PGC1a and $\beta$-actin. (F) Densitometry of PGC1a/ $\beta$-actin. Values are mean \pm standard deviation from three independent experiments and expressed as $n$-fold increase compared to untreated control set to 100. ANOVA followed by Tukey post-hoc test was used for statistical analysis. ${ }^{*} p<0.05 ;{ }^{\star \star} p<0.01$. The full-length gels are seen in the Supplementary Information.

\section{Supplementary Files}

This is a list of supplementary files associated with this preprint. Click to download.

- Supplementarylnformation.pdf

- Supplementarylnformation.pdf 